\title{
Modal Subordination in Type Theoretic Dynamic Logic
}

SaI QIAn, Institute of Energy, Jiangxi Academy of Sciences, China Philippe de Groote, INRIA Nancy Grand-Est, France Maxime Amblard, Université de Lorraine, LORIA, INRIA Nancy Grand-Est, CNRS, UMR 7503, France

\begin{abstract}
Classical theories of discourse semantics, such as Discourse Representation Theory (DRT), Dynamic Predicate Logic (DPL), predict that an indefinite noun phrase cannot serve as antecedent for an anaphor if the noun phrase is, but the anaphor is not, in the scope of a modal expression. However, this prediction meets with counterexamples. The phenomenon modal subordination is one of them. In general, modal subordination is concerned with more than two modalities, where the modality in subsequent sentences is interpreted in a context 'subordinate' to the one created by the first modal expression. In other words, subsequent sentences are interpreted as being conditional on the scenario introduced in the first sentence. One consequence is that the anaphoric potential of indefinites may extend beyond the standard limits of accessibility constraints.

This paper aims to give a formal interpretation on modal subordination. The theoretical backbone of the current work is Type Theoretic Dynamic Logic (TTDL), which is a Montagovian account of discourse semantics. Different from other dynamic theories, TTDL was built on classical mathematical and logical tools, such as $\lambda$-calculus and Church's theory of types. Hence it is completely compositional and does not suffer from the destructive assignment problem. We will review the basic set-up of TTDL and then present Kratzer's theory on natural language modality. After that, by integrating the notion of con-
\end{abstract}


versation background, in particular, the modal base usage, we offer an extension of TTDL (called Modal-TTDL, or M-TTDL in short) which properly deals with anaphora across modality. The formal relation between Modal-TTDL and TTDL will be discussed as well.

\section{Modal Subordination}

\subsection{Dynamic Semantics}

Around the middle of the last century, Alfred Tarski investigated the semantics of formal languages by defining the notion of truth (Tarski, 1944, 1956). Later on in the 1970s, his student Richard Montague established a model-theoretic semantics for natural language (Montague, 1970b a, 1973) by using the mathematic tools of that time, e.g., higherorder predicate logic, $\lambda$-calculus, type theory, intensional logic, etc. This work is known as the Montague Grammar (MG), which makes the possibility to interpret natural language, in particular English, as a formal language. Under MG, linguistic expressions are interpreted in terms of their contributions to the truth conditions of the sentences where they occur. This is recognized as the static view on meaning. Despite its prevailing influence in the field of logical semantics, MG was designed to account for the meaning of isolated sentences. Thus, linguistic phenomena that cross sentence boundaries, such as inter-sentential anaphora, donkey anaphora, presupposition, etc., lack proper explanations in MG.

Since the 1980s, in order to overcome the empirical problems arising from MG, a number of semantic theories have been established from the discourse perspective. Representative works include Discourse Representation Theory (DRT) (Kamp, 1981), File Change Semantics (FCS) (Heim, 1982), and Dynamic Predicate Logic (DPL) (Groenendijk and Stokhof, 1991). In contrast with classical logical semantics such as MG, these theories are subsumed under the label dynamic semantics, where the meaning of a linguistic expression is identified with its potential to change the context, rather than the truth conditions. More specifically, the meaning of a sentence is the change it brings about to an existing discourse where it occurs; the meaning of a non-sentential expression equals its contribution to that change. Summarized in a slogan, meaning is the "context change potential" (Heim, 1983). The notion of context in dynamic semantics denotes what gets changed during the interpretation. It is subject to the particular domain of research. For instance, when analyzing anaphoric relations between noun phrases and pronominal anaphors, the context resides in the discourse referents which have been introduced, namely the objects being talked about, and the possibilities that they are to be retrieved by anaphoric terms 
in subsequent discourse.

Per contrast to static semantics, the above mentioned dynamic theories manage to give an account for the inter-sentential anaphora and donkey anaphora. At the same time, they help to constrain a number of infelicitous anaphors as well. Take the following discourses:

(1) Bill doesn't have $\underline{\operatorname{a~car}}_{i} \cdot{ }^{*} \underline{\mathrm{It}}_{i}$ is black. (Karttunen, 1969)

(2) a. *Either Jones owns a bicycle $i$, or it $\underline{\text { it }}_{i}$ 's broken.

b. Jane either borrowed a car $_{i}$ or rented a truck $j$ to get to Boston. ${ }^{*} \underline{\mathrm{It}}_{i / j}$ broke down on the way. (Simons, 1996)

(3) Every $\operatorname{man}_{i}$ walks in the park. ${ }^{*} \underline{H e}_{i}$ whistles. (Groenendijk and Stokhof, 1991)

As indicated in each example, all anaphoric relations are problematic, and these anomalies can be correctly captured in dynamic semantics. According to the classical dynamic frameworks, negation blocks the accessibility of discourse referents within its scope, which explains example (1) disjunction blocks the accessibility of discourse referents from either disjunct, as well as from outside its scope, which explains example (2) and implication admits the accessibility of discourse referents in the antecedent from the consequent, but not from outside its scope, which explains example (3)

\subsection{Anaphora under Modality}

Although the well-established constraints in dynamic semantics can account for a wide range of empirical data concerning the accessibility of discourse anaphora, there are a number of exceptional linguistic examples, where the life-span of a discourse referent is longer than expected. The perspective of this section is to sketch one specific case: modal subordination, which is also the main problem that we are trying to investigate in the current paper.

At first glance, modality has a similar effect as negation in blocking discourse referent. That is to say, if an indefinite noun phrase (NP) occurs in the scope of some modal operator, e.g., must, can, shall, etc., its discourse referent cannot be anaphorically linked to expressions in subsequent discourse. For instance:

(4) a. You must write a letter $i$ to your parents. *They are expecting the letter . $_{\text {. }}$

b. Bill can make $\underline{a_{\text {kite }}}$. ${ }^{*}$ The kite $_{i}$ has a long string. (Karttunen, 1969)

In the above discourses, neither of the anaphoric expressions: the 
letter and the kite, can refer back to the corresponding indefinite NP. That is because both indefinites are located in the complement clauses governed by model auxiliaries, i.e., must in (4-a), can in (4-b). To account for examples as such in dynamic semantics, a basic strategy is to integrate two modal logic operators, namely the possibility operator $\diamond$ and the necessity operator $\square$, in the syntactic and semantic systems of the original theories. Modal operators are treated in a similar way as negation, which blocks discourse referents within its scope. We will not go further into this point since it is not the focus of the current paper.

Despite the observation drawn from example (4), the following data suggest that anaphoric references are not always impossible across modality.

(5) If John bought a book $\underline{b}_{i}$, he'll be home reading $\underline{i t}_{i}$ by now. $\underline{\mathrm{t}}_{i}{ }^{\prime} \mathrm{ll}$ be a murder mystery. (Roberts, 1989)

(6) A thief ${ }_{i}$ might break into the house. $\underline{\mathrm{He}}_{i}$ would take the silver. (Roberts, 1989)

In both above examples, the anaphoric expressions, namely the second it in (5) and he in (6), are interpreted as depending on the indefinites introduced under preceding modalities. These examples are different from (4) in the sense that the second sentences are not in the factual mood, rather, they contain modals of their own. Furthermore, in each case of (5) and (6) the modal in the second sentence is interpreted in a context 'subordinate' to that created by the first modal. In other words, successive non-factual discourse is interpreted as being conditional on the scenario introduced in the first sentence. Standard dynamic frameworks fail to give an explanation of examples of this sort no discourse referent can survive outside the scope of modal operators. This phenomenon, where the accessibility barriers assumed in classical dynamic semantics are broken down by continuous modals, is known as modal subordination (Roberts, 1987, 1989).

To account for such examples (5) and (6), Roberts combines Kratzer's theory of modality and DRT, where the blocked discourse referents are made available by repeating the whole sub-DRS into the DRS of the subordinate modal. However, Robert's approach is not completely satisfactory. On the one hand, although this combination is straightforward, it has to be accompanied with several constraints. Otherwise, this approach, namely accommodation of antecedent, is too powerful so it overgenerates and predicts that all referents introduced under modality will be accessible to subsequent discourse. On the other hand, the 
classical theory DRT is often criticized for lacking compositionality ${ }^{1}$. Furthermore, it suffers from the so-called destructive assignment problem. Hence, we will try to resolve the modal subordination problem based on a more recently proposed dynamic theory.

In the following section, we introduce the theoretical foundation of the current paper, namely Type Theoretic Dynamic Logic. In section 3 . we present some preliminary notions on natural language modality, as well as a now classical theory about modality in the linguistic perspective. Next, section 4 will focus on presenting our specific solution of modal subordination. Finally, in the last section we will delete out some general conclusions and further suggestions.

\section{Type Theoretic Dynamic Logic}

Around a decade ago, de Groote proposed a new dynamic framework Type Theoretic Dynamic Logic (TTDL) (de Groote, 2006). This framework aims to study the semantics of sentences and discourses in a uniform and classical way. In order to achieve dynamics, TTDL integrates the notion of left and right contexts into MG: given a sentence, its left context denotes the discourse that precedes it, namely what has already been processed; its right context is the continuation (Strachey and Wadsworth, 1974), denoting the discourse that follows it, namely what will be processed in future. A sentence is interpreted with respect to both its left and right contexts, and its semantics is abstracted over the two contexts.

Technically, TTDL sticks to the tradition of MG. It only makes use of standard mathematical and logical tools, such as $\lambda$-calculus and theory of types. Logical notions such as free and bound variables, quantifier scopes, are as usual. And the only operations involved are standard $\alpha$ conversions and $\beta$-reductions. This property enables it to inherit all nice properties of well-established mathematics and logics. In what follows, we present the formal details of TTDL.

Akin to other systems based on the simply typed $\lambda$-calculus, the syntax of TTDL can be defined in terms of the notion of higher order signature (de Groote, 2001), which is a triplet consisting of a finite set of atomic types, a finite set of constant symbols, and a function that assigns each constant a type.

Definition 2.1. The signature of TTDL, in notation $\Sigma_{T T D L}$, is defined

\footnotetext{
${ }^{1}$ The notion of compositionality has been successfully integrated in some later versions of DRT.
} 
as follows:

$$
\begin{aligned}
\Sigma_{T T D L}=\langle & \{\iota, o, \gamma\}, \\
& \left\{\top, \wedge, \neg, \exists, \_::_{-}, \text {sel, nil }\right\}, \\
& \{\top: o, \wedge: o \rightarrow o \rightarrow o, \neg: o \rightarrow o, \\
& \exists:(\iota \rightarrow o) \rightarrow o,{ }_{-}::_{-}: \iota \rightarrow \gamma \rightarrow \gamma, \\
& \text { sel }: \gamma \rightarrow \iota, \text { nil }: \gamma\}\rangle
\end{aligned}
$$

Logical constants such as $\top$ (tautology), $\wedge$ (conjunction), $\neg$ (negation) and $\exists$ (existential quantifier) are exactly the same as in First Order Logic (FOL). However, besides the two atomic types in Church's simple type theory (Church, 1940) $: \iota$ denoting the type of individuals and $o$ denoting the type of propositions, there is a third one in TTDL: $\gamma$ denoting the type of left contexts. The right context, which is interpreted as the continuation of the sentence, is a function from left contexts to truth values. So its type is $\gamma \rightarrow o$. For instance, assume $e$ is a left-context variable, then the empty right context can be defined in the compact term stop as follows:

$$
\text { stop } \triangleq \lambda e . \top
$$

In order to solve pronominal anaphora in TTDL, the left context is modeled as a list of individuals. This explains the type of the empty context "nil" in $\Sigma_{T T D L}$. In addition to that, two operators are introduced in definition 2.1. The first is the list constructor "::". Its function is to add new individuals (of type $\iota$ ) into existing contexts (of type $\gamma$ ). Hence the type of "::" is $\iota \rightarrow \gamma \rightarrow \gamma$. The second is the choice operator "sel", it takes a left context (of type $\gamma$ ) as argument and yields back an individual (of type $\iota$ ). Hence its type is $\gamma \rightarrow \iota$.

In standard truth-conditional semantics, a sentence expresses a proposition, which is of type $o$. While in TTDL, a sentence will be interpreted with respect to both its left and right contexts, which are of type $\gamma$ and $\gamma \rightarrow o$, respectively. If we use $s$ to denote the syntactic category of sentences, $\llbracket \rrbracket_{T T D L}$ to denote the semantic interpretation under TTDL, then:

$$
\llbracket s \rrbracket_{T T D L}=\gamma \rightarrow(\gamma \rightarrow o) \rightarrow o
$$

Discourses, which also express propositions, are interpreted in the same way as single sentences. So, letting $d$ be the syntactic category of discourses, we have:

$$
\llbracket d \rrbracket_{T T D L}=\gamma \rightarrow(\gamma \rightarrow o) \rightarrow o
$$

In order to contrast with $o$, which is the type of (standard/static) propositions, we call $\gamma \rightarrow(\gamma \rightarrow o) \rightarrow o$ the type of dynamic proposi- 
tions. Hereinafter, we will use $\Omega$ as an abbreviation for $\gamma \rightarrow(\gamma \rightarrow o) \rightarrow$ $o$, namely:

$$
\Omega \triangleq \gamma \rightarrow(\gamma \rightarrow o) \rightarrow o
$$

After presenting the typing information in TTDL, let us proceed to the logics of the framework. Let $A$ and $B$ be variables denoting dynamic propositions, $e$ and $e^{\prime}$ be variables denoting left contexts, $\phi$ be a variable denoting the right context, then the dynamic conjunction $\wedge_{T T D L}^{d}$ in TTDL, which conjoins two dynamic propositions, is defined as follow 2 .

$$
\wedge_{T T D L}^{d} \triangleq \lambda A B e \phi . A e\left(\lambda e^{\prime} . B e^{\prime} \phi\right)
$$

In the above formula, $e$ and $\phi$ are the left and right contexts of the conjunction, they are also called the current left and right contexts. Formula 2.5 can be further elaborated as follows. First of all, the semantics of a conjunction is contributed by both conjuncts, this explains why $A$ and $B$ are both involved in the composition. In addition, the left context of the first conjunct is the current left context, this is why $e$ is passed to $A$; the right context of the second conjunct is the current right context, this is why $\phi$ is passed to $B$. Finally, the right context of the first conjunct is made up of the second conjunct and the current right context, this explains why $\lambda e^{\prime} \cdot B e^{\prime} \phi$ is passed to $A$; the left context of the second conjunct is made up of the first conjunct and the current left context, this explains why $e^{\prime}$, which forms a $\lambda$-abstraction and will be substituted by a complex structure of type $\gamma$ (consisting of $e$ and information in $A$ ), is passed to $B$.

In order to negate dynamic propositions, TTDL defines the dynamic negation operator $\neg_{T T D L}^{d}$ as follows:

$$
\neg_{T T D L}^{d} \triangleq \lambda A e \phi . \neg(A \text { e stop }) \wedge \phi e
$$

where stop was defined in formula 2.1. The operator $\neg_{T T D L}^{d}$ takes a dynamic proposition $A$ and returns its dynamically negated counterpart, hence it is of type $\Omega \rightarrow \Omega$. The right hand side of formula 2.6 can be further understood as follows. Firstly, the left context of the to-be-negated proposition $A$ is the current left context, this is why $e$ is passed to $A$. Furthermore, we do not want negation to take scope over any future part of the discourse, so the empty right context stop, rather than the current right context $\phi$, is passed to $A$. Finally, a dynamic negation does not have the potential to update the left context, this is why $\phi e$, the function-application of the original left and right contexts, appears as a conjunct at the end of the formula.

\footnotetext{
${ }^{2}$ The conjunction we define here is for propositions only, it is hence not related in terms of semantics with respect to other categories, e.g., NP, VP.
} 
As to the dynamic existential quantifier in TTDL, it is defined as:

$$
\exists_{T T D L}^{d} \triangleq \lambda \operatorname{Pe} \phi . \exists(\lambda x . P x(x:: e) \phi)
$$

The dynamic quantifier $\exists_{T T D L}^{d}$ takes a dynamic property $P$ of type $\iota \rightarrow \Omega$, and returns a existentially quantified dynamic proposition. Hence the semantic type of the operator $\exists_{T T D L}^{d}$ is $(\iota \rightarrow \Omega) \rightarrow \Omega$. The right hand side of formula 2.7 can be understood as follows. In an existentially quantified dynamic proposition, variables which are bound by the existential quantifier shall update the current left context, this is why the updated context $(x:: e)$ is passed to the proposition within the scope of $\exists$.

Above we have presented the dynamic logic in TTDL, in particular, the definitions of the dynamic operators. In fact, there exists a systematic translation, which associates (standard/static) logical expressions to their dynamic counterparts. The translation process is concerned with both types and $\lambda$-terms, which will be examined one by one below.

Notation 2.1. We use the bar notation, for instance, $\bar{\tau}$ or $\bar{M}$, to denote the dynamic translation of a type $\tau$ or a $\lambda$-term $M$ in TTDL.

Definition 2.2. The dynamic translation of a type $\tau$ : $\bar{\tau}$, is defined inductively as follows:

1. $\bar{\iota}=\iota$;

2. $\bar{o}=\Omega$;

3. $\overline{\sigma \rightarrow \tau}=\bar{\sigma} \rightarrow \bar{\tau}$, where $\tau$ and $\sigma$ are types.

According to definition 2.2 the static and dynamic types of individuals are both $\iota$, while the static and dynamic type of propositions are $o$ and $\Omega$, respectively. The dynamic translation of a function type is still a function type, with the argument type and the result type being translated respectively.

The dynamic translation of $\lambda$-terms will ground on the following two functions: the dynamization function $\mathbb{D}$ and the staticization function $\mathbb{S}$, whose definitions are mutually dependent. They will be used to translate non-logical constants.

Definition 2.3. The dynamization function $\mathbb{D}_{\tau}$, which takes an input $\lambda$-term $A$ of type $(\gamma \rightarrow \tau)$, returns an output $\lambda$-term $A^{\prime}$ of type $\bar{\tau}$; the staticization function $\mathbb{S}_{\tau}$, which takes an input $\lambda$-term $A^{\prime}$ of type $\bar{\tau}$, returns an output $\lambda$-term $A$ of type $(\gamma \rightarrow \tau)$.

$\mathbb{D}_{\tau}$ is defined inductively on type $\tau$ as follows:

1. $\mathbb{D}_{\iota} A=A$ nil;

2. $\mathbb{D}_{o} A=\lambda e \phi .(A e \wedge \phi e)$; 


\section{3. $\mathbb{D}_{\alpha \rightarrow \beta} A=\lambda x \cdot \mathbb{D}_{\beta}\left(\lambda e \cdot A e\left(\mathbb{S}_{\alpha} x e\right)\right)$.}

$\mathbb{S}_{\tau}$ is defined inductively on type $\tau$ as follows:

1. $\mathbb{S}_{\iota} A^{\prime}=\lambda e \cdot A^{\prime}$

2. $\mathbb{S}_{o} A^{\prime}=\lambda e \cdot A^{\prime}$ e stop;

3. $\mathbb{S}_{\alpha \rightarrow \beta} A^{\prime}=\lambda e \cdot\left(\lambda x \cdot \mathbb{S}_{\beta}\left(A^{\prime}\left(\mathbb{D}_{\alpha}\left(\lambda e^{\prime} \cdot x\right)\right)\right) e\right)$.

Now based on definition 2.3 , we can proceed to the dynamic translation of $\lambda$-terms.

Definition 2.4. The dynamic translation of a $\lambda$-term $M$ (of type $\tau$ ): $\bar{M}$, which is another $\lambda$-term of type $\bar{\tau}$, is defined as follows:

1. $\bar{x}=x$, if $x$ is a variable;

2. $\overline{\mathbf{a}}=\mathbb{D}_{\tau}(\lambda e \cdot \mathbf{a})$, if $\mathbf{a}$ is a non-logical constant and $\mathbf{a}: \tau$;

3. $\bar{\wedge}=\wedge_{T T D L}^{d}$, see formula 2.5 .

4. $\overline{\bar{\neg}}=\neg_{T T D L}^{d}$, see formula 2.6 .

$5 . \bar{\exists}=\exists_{T T D L}^{d}$, see formula 2.7 .

6. $\overline{(M N)}=(\bar{M} \bar{N})$;

7. $\overline{(\lambda x \cdot M)}=(\lambda x \cdot \bar{M})$.

The dynamic counterparts of the derived operators, such as $\vee$ (disjunction), $\rightarrow$ (implication), and $\forall$ (universal quantifier), are defined in terms of primitive logical constants and the corresponding rules in definition 2.4. Since the semantics of TTDL is almost the same as the one of FOL, we will not dig into that. Illustrations of TTDL will not be presented here for reasons of space. For more examples, please refer to (de Groote, 2006) and (Lebedeva, 2012).

With the above set-up, TTDL manifests the same empirical coverage on discourse anaphora as other dynamic frameworks, such as DRT and DPL. In the following section, we will first discuss modality in more detail from the linguistic perspective. Then we will present the theory of modality developed by Angelika Kratzer (Kratzer, 1977, 1981, 1986, 1991). After that in section 4. we shall combine Kratzer's theory of modality with TTDL, yielding an adaptation of TTDL called Modal TTDL (M-TTDL), which treats the modal subordination problem in traditional montagovian style. A formal link between the new adaptation and TTDL will be established as well.

\section{Preliminary Notions on Modality}

\subsection{Modality in Natural Language Semantics}

Generally speaking, modality is a semantic notion which is concerned with possibility and necessity. In linguistics, modality enables people to talk about things beyond the actual here and now (von Fintel, 2006). 
It is reflected in the set of phenomena that express notions such as belief, attitude and obligation in natural language sentences. Modality, which has been pervasively attested across almost all languages, can be established by a wide range of grammatical categories and constructions. Take English for example, there are modal auxiliaries (e.g., must, may, should, might), modal adjective and adverbs (e.g., it is possible ..., possibly, necessarily, probably), conditionals (e.g., if ... then ...), propositional attitude verbs (e.g., believe, know, hope), etc.

One aspect of the semantics of modality is modal force, namely the strength of a modal, i.e., possibility and necessity. The two corresponding operators are treated as quantifiers ranging over possible worlds: $\diamond$ as existential, $\square$ as universal. Because of that, possibility and necessity are also called existential force and universal force (respectively). The force of a modal expression is inherently contained in its lexical meaning. For instance, modals such as may, might and could always denote a possibility; while modals such as must, should and would always denote a necessity one.

Another aspect on the semantics of modality is modal flavor, it indicates the particular sort of premise information, e.g., epistemic, deontic, etc., with respect to which a modal is interpreted. This notion is motivated by the fact that it is insufficient to interpret modal expressions only relative to their modal forces. According to modal flavor, modalities can be classified into different sub-types. Let's take the following sentences for example, where the modal is considered to be ambiguous:

a. All Maori children must learn the names of their ancestors.

b. The ancestors of the Maoris must have arrived from Tahiti. (Kratzer, 1977)

Both (7-a) and (7-b) contain the same modal must, so each of them expresses a universal force. However, the meaning of must varies from one sentence to another. For instance, in (7-a), the modal must refers to an obligation or a duty that the Maori children should obey or fulfill, it is called a deontic modality; in (7-b) the same modal denotes some knowledge or belief, it is called an epistemic modality. This distinction can be revealed in an explicit way by paraphrasing (7) as follows, where an in view of ... adverbial phrase is added at the beginning of each sentence:

(8) a. In view of what their tribal duties are, the Maori children must learn the names of their ancestors.

b. In view of what is known, the ancestors of the Maoris must 
have arrived from Tahiti. (Kratzer, 1977)

The modal must in (7-a) means "necessary in view of what their tribal duties are"; while must in (7-b) means "necessary in view of what is known". A similar contrast can be found in the following examples:

(9) a. According to his dating coach, John must dance at parties.

b. Since John hangs out with Linda at parties, he must dance at parties. (Starr, 2012)

By only looking at the modalized sentence John/he must dance at parties, which is shared by both discourses in (9), we are not able to tell whether it refers to an obligation (deontic), or a piece of knowledge (epistemic), or maybe something else. However, with the help of the prefixed adverbial phrases in (9), we can unambiguously determine that the shared modalized sentence expresses a deontic modality in (9-a) while it expresses an epistemic one in (9-a).

Actually, besides the deontic and epistemic modality as we have shown in the above examples, there are also other types of modality that a modal expression can express, such as bouletic (wishes or desires), teleological (goals), circumstantial (circumstances), etc., all of which are called the flavor of a moda ${ }^{3}$. For instance, all the following examples involve the same modal expression have to, but denotes different modalities:
a. It has to be raining. [after observing people coming inside with wet umbrellas; epistemic modality]
b. Visitors have to leave by six pm. [hospital regulations; de- ontic]
c. You have to go to bed in ten minutes. [stern father; bouletic]
d. I have to sneeze. [given the current state of one's nose; circumstantial]
e. To get home in time, you have to take a taxi. [telelological] (von Fintel, 2006)

For more examples, please refer to (Kratzer, 1977, Portner, 2009). Different from the modal force, which solely comes from the lexical meaning of a modal, the modal flavor depends on the specific situation where the modal is applied. Sometimes, it is given by linguistic means, where there are noticeable indicators such as the adverbial phrases in view of $\ldots$ and according to ... in (8) and (9) most of the time however,

\footnotetext{
${ }^{3}$ The names of these different flavors may vary from author to author.
} 
no indicators are explicitly presented and the readers have to resolve the most appropriate flavor based on clues from the context of use, for instance, as in (7) and (10).

In order to interpret modal expressions in formal systems such as Modal Predicate Logic (MPL), we need to correctly handle both above mentioned semantic aspects. The treatment of modal force is relatively straightforward: $\diamond$ is the existential quantifier over possible worlds, $\square$ is the universal quantifier over possible worlds. As to modal flavor, what we can do is to assign each different modal a different set of possible worlds which it quantifies over. In other words, to associate each modal a corresponding accessibility relation 4 . However, from a generalization point of view, this strategy is not satisfactory. In the next section, we will sketch Kratzer's theory on modality, which aims to give a unified analysis on different types of modality (e.g., epistemic, deontic, bouletic, etc.).

\subsection{Kratzer's Theory of Modality}

Currently, Kratzer's theory of modality (Kratzer, 1977, 1981, 1986, 1991 ) is the most studied work in this field. It has served as the foundation for a large number of subsequent works on modality. One of the most essential motivations of Kratzer is to tackle the problem of lexical ambiguity among modals, providing a uniform treatment to modals of various modal forces.

In her theory, Kratzer proposes that modals are context-dependent, rather than ambiguous between various flavors. As we mentioned before, the must in examples (7-a) and (7-b) means "necessary in view of what their tribal duties are" and "necessary in view of what is known", respectively. However, if we understood modals in this way, the adverbial phrases as in examples (8) and (9) would be redundant, since modals would carry all the necessary information, while this is not the case. So Kratzer's strategy is to make a clear-cut division between the two aspects of modal semantics that we presented above, that is to say, the force of a modal exhausts its meaning. As to the flavor, which is not part of the meaning of a modal any more, it is fixed by the context. We will explain this in more detail below.

A modal sentence, as far as Kratzer concerns, is interpreted in a modular way such that it consists of three parts: a neutral modal operator, a background context, and a proposition under discussion. The last parameter is relatively easy to understand, it is the proposition

\footnotetext{
${ }^{4}$ The accessibility relation is a binary relation in possible world semantics Kripke 1959, 1963), denoting the possibility to reach a possible world from another.
} 
governed by the corresponding modal operator. The modal operator, which is uniquely determined by the modal expression, is neutral in the sense that it only denotes the modal force, namely, whether it is existential or universal. The background context is the foundation for the uniform interpretation of various types of modality. It indicates the particular flavor that a modal is applied to. In other words, it restricts the domain of worlds which modal operators quantify over.

In order to model the background information, Kratzer proposes the notion of conversational background. Generally speaking, a conversational background stands for the entity denoted by adverbial phrases such as in view of and according to. It provides a particular premise, with respect to which a modal sentence will be evaluated. This premise can be formalized as a set of propositions (knowledges or obligations), and it is sensitive to the world. For instance, take the epistemic conversational background in view of what is known in (7-b) it gives a set of propositions known at the utterance world, which are different from world to world (people may know different things in different world). Analogously, take the deontic conversational background according to his dating coach in (9-a) for example, it supplies a set of commands from the coach that John should follow, which also differ from world to world. We formalize conversational background as follows:

Definition 3.1. A conversational background is a function from possible worlds to sets of (modal) propositions.

For instance, assume $f$ is a conversational background, $W$ is a set of possible worlds, $w \in W$ is a possible world, then $f(w)=\left\{\phi_{1}, \phi_{2}, \ldots\right\}$ is a set of propositions which contributes the background information at $w$. In other words, all propositions in $f(w)$, namely $\phi_{1}, \phi_{2}, \ldots$, are necessarily true $e^{5}$ at $w$. The notion of conversational background closely correlates to the accessibility relation in possible world semantics. In fact, the former can be used in place of the latter for defining the semantics of modals. Please refer to (Portner, 2009) for more details.

In sum, Kratzer's theory as we have presented so far, is a contextualized version of the standard modal logic such as MPL, it is called the relative modality. Different readings of a modal expression are reduced to the specification of a single modal force, together with various context-dependent conversational backgrounds. Hence we are able to interpret modals in a uniquely unambiguous way. Also, correlated notions such as the accessibility relation, together with its properties, can be recast in terms of conversational background correspondingly.

\footnotetext{
${ }^{5}$ Whether $\phi_{1}, \phi_{2}, \ldots$ are knowledges, or obligations, or goals, depends on the particular type of the conversational background $f$.
} 
However, in natural language, modalities are not divided by a neat dichotomy. Here are some specific linguistic examples:
a. It is barely possible to climb Mount Everest without oxy- gen.
b. It is easily possible to climb Mount Toby.
c. They are more likely to climb the West Ridge than the Southeast Face.
d. It would be more desirable to climb the West Ridge by the Direct Route. (Kratzer, 1991)

In relative modality, possibility is defined as an absolute concept. However, in order to account for example (11) we need to tune modality in a scalable fashion. Hence, Kratzer proposes that modal expressions should be interpreted with respect to two conversational backgrounds: one, as we introduced above, is called the modal base, it provides the background information, namely a set of accessible worlds; the other is called the ordering source, which imposes an ordering on the accessible worlds, i.e., some worlds are more accessible than others. This machinery will not only resolve the problem of graded modality, but also cope with a series of other modality-related problems (Kratzer, 1991. Schoubye, 2011), such as the inconsistencies, conditionals, etc. In this paper, we will sidestep the ordering source, and only consider the modal base usage of conversational background. Interested readers may refer back to the original reference for more information (Kratzer. 1981).

\section{Modal Subordination under TTDL}

In this section, we will integrate epistemic modality within the continuationbased dynamic framework TTDL as introduced earlier in section 2, we will call the new framework Modal TTDL (M-TTDL). As explained in section 3.2 , a conversational background is a function from possible worlds to sets of propositions, which are the ones that are necessarily true at the given world. They serve as the common ground information, or premise assumption, for subsequent modally subordinated utterances. Hence our strategy for achieving M-TTDL is to enrich the context of TTDL with the notion of conversational background (Kratzer, 1981), in particular the modal base.

In the following, we will first present the formal framework, including the particular signature for M-TTDL, and the typing information and the way in which (modal) proposition, left context, right context, etc., are respectively interpreted; then we will define some preliminary 
functions that facilitate later presentation; after that, we propose the formal framework, including the syntax and semantics; finally, the lexical entries, together with the treatments of some puzzling examples will be provided.

\subsection{Formal Framework}

As its ancestor system TTDL, the adaptation M-TTDL is a framework based on the simply typed $\lambda$-calculus. For all the formal details, please refer back to section 2. Below, we specify the signature of M-TTDL in detail.

Since M-TTDL is concerned with the notion of possible world, which is missing in TTDL, we need a different signature from the previous one (see definition 2.1). Types and constants that are correlated with possible worlds need to be incorporated in M-TTDL. As a result, we keep the two conventional ground types in M-TTDL: $\iota$ for individuals, and $o$ for truth values. Besides, a third primitive type $s$ is employed for possible worlds. As to $\gamma$, which is the type denoting lists of discourse referents, is abandoned because the context in M-TTDL will contain propositions (the modal base) rather than variables. In the following, we provide a formal characterization of the new signature. Please note that only the types of logical constants are specified. The particular type of a non-logical constant will be indicated when it is employed.

Definition 4.1. The signature $\Sigma_{M-T T D L}$ is defined as follows:

$$
\begin{aligned}
\Sigma_{M-T T D L}=\langle & \{\iota, o, s\},\left\{\top, \wedge, \neg,{ }^{\iota} \exists,{ }^{s} \exists, \text { sel }, \mathbf{H}\right\}, \\
& \left\{\top: o, \wedge: o \rightarrow o \rightarrow o, \neg: o \rightarrow o,{ }^{\iota} \exists:(\iota \rightarrow o) \rightarrow o,\right. \\
& s \exists:(s \rightarrow o) \rightarrow o, \text { sel }:(\iota \rightarrow o) \rightarrow o \rightarrow \iota, \mathbf{H}: s\}\rangle
\end{aligned}
$$

Now let's take a close look at the logical constants. In the first place, we abandon in $\Sigma_{M-T T D L}$ the familiar list constructor _::- and the empty list of referents nil, because the left context in M-TTDL is made up of propositions (the modal base), rather than variables. In addition, with respect to the modification on the left context, the choice operator sel is changed accordingly. In previous systems, it is used to pick up a variable from a list of referents (of type $\gamma \rightarrow \iota$ ). But in M-TTDL, it will do the same job with respect to an input property (of type $\iota \rightarrow o$ ) and the current modal base (of type $o$ ). The former is the criterion based on which sel makes its decision. This explains the semantic type of sel as defined in $\Sigma_{M-T T D L}$. Furthermore, we distinguish between the quantifier over individuals ${ }^{\iota} \exists$ and the one over possible worlds ${ }^{s} \exists$. Their difference is revealed in their corresponding types. Some other conventional logical constants, such as $\rightarrow$ (implication), $\vee$ (disjunction), $\forall$ (universal quantifier), are defined same as before in terms of the above 
primitives. Please note that corresponding to the two existential quantifiers, there are also a pair of universal quantifiers: ${ }^{\iota} \forall$ and ${ }^{s} \forall$, the former ranges over individual variables, the latter over possible world variables. Finally, the possible world constant $\mathbf{H}$ denotes the current world. It will be used to provide the world of evaluation at the end of the semantic interpretation.

For the rest of this subsection, we will focus on the typing information in M-TTDL. The way to interpret left context, right context, and propositions will be elucidated sequentially. As we mentioned above, $\iota$ and $o$ are still the types for individuals and truth values, respectively. However in modal systems, such as MPL, a (modal) proposition is interpreted as a set of possible worlds, rather than a truth value. Hence its type should be $s \rightarrow o$. Hereinafter, we abbreviate it as $o_{i}$, namely:

$$
o_{i} \triangleq s \rightarrow o
$$

Correspondingly, the semantic type of 1-place predicates, such as man and walk_in, is updated to $\iota \rightarrow o_{i}$; the type of 2-place predicates, such as beat and eat, is updated to $\iota \rightarrow \iota \rightarrow o_{i}$.

To explain the interpretation of the left context, we first propose the concept of environment. It is an ordered pair consisting of two modal propositions: the background information and the base information. The purpose of an environment is twofold: on the one hand, it encodes the propositions necessarily true at the given world, which is the background information; on the other hand, it enables to pass updated propositions from a possible world to accessible ones, which is the base information. Both the background and the base are propositions, they are hence of type $o_{i}$. As a result, the type of an environment is $\left(o_{i} \times o_{i}\right)$. If we use $T_{e n v}, T_{b k}$, and $T_{b a}$ to denote the type of environment, background, and base, respectively, we can draw the following formulas:

$$
\begin{gathered}
T_{b k} \triangleq o_{i} \\
T_{b a} \triangleq o_{i} \\
T_{e n v} \triangleq T_{b k} \times T_{b a}=o_{i} \times o_{i}
\end{gathered}
$$

Based upon the notion of environment, we thus define another concept: generalized environment, which is in parallel with the conversational background in Kratzer's theory. As we know, the conversational background is a function from possible worlds to sets of propositions (or equivalently, the conjunction consisting of all propositions). Analogously, the generalized environment is a mapping from possible worlds to environments. This means that, if we apply a generalized environment to a particular world, it will yield the environment at that 
world. Consequently, if we use $T_{g e n v}$ to denote the type of generalized environments, it can be represented as follows:

$$
T_{g e n v} \triangleq s \rightarrow T_{e n v}
$$

In fact, the generalized environment can be regarded as an enhanced version of the conversational background. By applying it to a possible world argument, we obtain a pair of (modal) propositions. The first element, namely the background proposition, is exactly equivalent to the current modal base: it is the conjunction of all propositions that are necessarily satisfied/recognized at that possible world. And the background can be incrementally updated during the discourse processing, when new logical contents/propositions which are necessarily true in that world are provided. The second element of the pair, namely the base proposition, serves as a "buffer": appearing in the form of a conjunction as well, it consists of the propositions to be updated to accessible worlds. Its content will be reset after the updating in order to avoid information duplication. An illustration will be provided in section 4.6 .

Besides environment and generalized environment, we need to introduce the concept of the salient world, or equivalently, the world of interest, for the process of discourse incrementation. Its purpose is to record the current position of the processing in the overall possible worlds hierarchy, this will determine in which world the propositions expressed by subsequent utterances are to be integrated. Note that this is different from the world of evaluation (the world where the sentence is uttered) in possible world systems such as MPL. The distinction of the two concepts can be illustrated as follows. When we say "it might rain tomorrow", the world of utterance, namely the current world, is the world of evaluation, while the salient world will be an accessible world of the current world where it rains tomorrow. If we continue with "the flight might be canceled", the world of evaluation remains unchanged, while the salient world switches to an accessible world, where the flight is canceled, of the previous salient world.

With the above notions, we establish the left context in M-TTDL by encapsulating the salient world and the generalized environment in an ordered pair. By convention, we use $\gamma_{i}$ to symbolize the type of left context, then:

$$
\gamma_{i} \triangleq s \times T_{g e n v}
$$

If we unfold $\gamma_{i}$ with all primitive types, we will obtain the following 
typing information:

$$
\begin{aligned}
\gamma_{i} & =s \times\left(s \rightarrow T_{\text {env }}\right) \\
& =s \times\left(s \rightarrow\left(o_{i} \times o_{i}\right)\right) \\
& =s \times(s \rightarrow((s \rightarrow o) \times(s \rightarrow o)))
\end{aligned}
$$

Same as in TTDL, the right context in M-TTDL is interpreted as a function from left contexts to (modal) propositions, hence its semantic type is $\gamma_{i} \rightarrow o_{i}$. Similarly, if we unfold it, we will obtain:

$$
\begin{aligned}
\gamma_{i} \rightarrow o_{i} & =\left(s \times T_{\text {genv }}\right) \rightarrow o_{i} \\
& =\left(s \times\left(s \rightarrow T_{\text {env }}\right)\right) \rightarrow o_{i} \\
& =\left(s \times\left(s \rightarrow\left(o_{i} \times o_{i}\right)\right)\right) \rightarrow o_{i} \\
& =(s \times(s \rightarrow((s \rightarrow o) \times(s \rightarrow o)))) \rightarrow(s \rightarrow o)
\end{aligned}
$$

Accordingly, a dynamic proposition in M-TTDL is interpreted as a function which takes a left context and a right context, and returns a (modal) proposition. Both sentences and discourses will be treated in the same manner. Assume $s$ and $d$ are syntactic categories of sentences and discourses, respectively, then:

$$
\begin{aligned}
& \llbracket s \rrbracket=\gamma_{i} \rightarrow\left(\gamma_{i} \rightarrow o_{i}\right) \rightarrow o_{i} \\
& \llbracket d \rrbracket=\gamma_{i} \rightarrow\left(\gamma_{i} \rightarrow o_{i}\right) \rightarrow o_{i}
\end{aligned}
$$

Again, we abbreviate the complex type with a compact term $\Omega_{i}$, namely:

$$
\Omega_{i} \triangleq \gamma_{i} \rightarrow\left(\gamma_{i} \rightarrow o_{i}\right) \rightarrow o_{i}
$$

By unfolding formula 4.6, we can obtain the following result:

$$
\begin{aligned}
\Omega_{i}= & \gamma_{i} \rightarrow\left(\gamma_{i} \rightarrow o_{i}\right) \rightarrow o_{i} \\
= & \left(s \times T_{\text {genv }}\right) \rightarrow\left(\left(s \times T_{\text {genv }}\right) \rightarrow o_{i}\right) \rightarrow o_{i} \\
= & \left(s \times\left(s \rightarrow T_{\text {env }}\right)\right) \rightarrow\left(\left(s \times\left(s \rightarrow T_{\text {env }}\right)\right) \rightarrow o_{i}\right) \rightarrow o_{i} \\
= & (s \times(s \rightarrow((s \rightarrow o) \times(s \rightarrow o)))) \rightarrow \\
& ((s \times(s \rightarrow((s \rightarrow o) \times(s \rightarrow o)))) \rightarrow(s \rightarrow o)) \rightarrow \\
& (s \rightarrow o)
\end{aligned}
$$

As we may observe from formula 4.7, the type of dynamic propositions in M-TTDL is rather complicated, particularly it involves a number of occurrences of possible worlds (of type $s$ ) in different positions. However, by looking at the folded form, i.e., formula 4.6, it is clearly a member of the continuation semantic family.

Up until now, we have presented the typing information in M-TTDL. In what follows, we will first introduce some functions which are con- 
cerned with the modal base, possible worlds, and correlated concepts. They are cornerstones for our future presentation. Afterwards, we will provide the dynamic logic in M-TTDL, as well as the systematic dynamic translation.

\subsection{Elementary Functions}

In this subsection, we will introduce some fundamental functions which are concerned with the above introduced concepts such as environment, generalized environment, context, etc. These functions shall be presented in various groups, based on the particular semantic object they are working on. They will largely be used to construct lexical entries, which we will see in the succeeding subsection.

To save space, we will not elaborate each function. Instead, more details on the elementary functions can be found in section 1 of the appendix.

\section{Modalized Logical Constants}

First of all, let's have a look at a set of modalized logical constants, which are defined in terms of the constants in the signature $\Sigma_{M \text {-TTDL }}$ (definition 4.1). These terms are proposed for the sake of saving space, and they will provide a better readability in subsequent function definitions.

- Modal conjunction $o_{i} \rightarrow o_{i} \rightarrow o_{i}$

$$
\wedge_{i} \triangleq \lambda A B i .(A i \wedge B i)
$$

- Modal negation: $o_{i} \rightarrow o_{i}$

$$
\neg i \triangleq \lambda A i \cdot \neg(A i)
$$

- Modal existential quantifier for individuals: $\left(\iota \rightarrow o_{i}\right) \rightarrow o_{i}$

$$
{ }^{\iota} \exists_{i} \triangleq \lambda P i \cdot{ }^{\iota} \exists(\lambda x . P x i)
$$

- Modal tautology: $o_{i}$

$$
\top_{i} \triangleq \lambda i . \top
$$

\section{Environment and Salient World Manipulation}

After the functions on modal propositions, let's turn to the ones which deal with salient world and environment.

- Retrieve the salient world: $\gamma_{i} \rightarrow s$

$$
\text { woi } \triangleq \lambda e . \pi_{1} e
$$

\footnotetext{
${ }^{6}$ This is the description of the function, which is followed by its corresponding semantic type. In subsequent function introductions, we will stick to the same notation.
} 
- Retrieve the generalized environment: $\gamma_{i} \rightarrow T_{\text {genv }}$

$$
\text { genv } \triangleq \lambda e . \pi_{2} e
$$

- Retrieve the environment: $\gamma_{i} \rightarrow s \rightarrow T_{\text {env }}$

$$
\mathbf{e n v} \triangleq \lambda e i .(\text { genv } e i)
$$

- Modify the salient world: $\gamma_{i} \rightarrow s \rightarrow \gamma_{i}$

$$
\text { change_woi } \triangleq \lambda e i .\langle i,(\text { genv } e)\rangle
$$

- Retrieve the background: $\gamma_{i} \rightarrow s \rightarrow o_{i}$

$$
\mathbf{b k g d} \triangleq \lambda e i . \pi_{1}(\mathbf{e n v} e i)
$$

- Retrieve the base: $\gamma_{i} \rightarrow s \rightarrow o_{i}$

$$
\text { base } \triangleq \lambda e i . \pi_{2}(\mathbf{e n v} e i)
$$

\section{Context Manipulation}

In this subsection, we will have a look at the functions which manipulate generalized environments and contexts. First, we define the following notation:

Definition 4.2. Let $w, w^{\prime} \in W$ be possible worlds, $G$ a generalized environment, $E$ an environment, $\mathbf{R}$ the accessibility relation. The notation $G[w:=E]$ stands for a generalized environment such that:

$$
G[w:=E]\left(w^{\prime}\right)= \begin{cases}E & \text { if } \mathbf{R}\left(w, w^{\prime}\right), \\ G\left(w^{\prime}\right) & \text { otherwise. }\end{cases}
$$

As indicated in definition $4.2, G[w:=E]$ is itself a generalized environment, whose interpretation relies on the input possible world argument. If the input world is accessible to $w$, then environment $E$ will be returned, otherwise, the generalized environment $G$ is applied to the input world. The following functions will be presented based on the above notation.

- Update the generalized environment: $T_{g e n v} \rightarrow s \rightarrow T_{e n v} \rightarrow T_{g e n v}$

$$
\text { up_genv } \triangleq \lambda G i E . G[i:=E]
$$

- Update the left context: $\gamma_{i} \rightarrow s \rightarrow o_{i} \rightarrow \gamma_{i}$

$$
\begin{aligned}
\text { up_context } \triangleq \lambda e i A .\langle( & \text { woi } e), \\
& \text { up_genv } \\
& (\text { genv } e) \\
& i \\
& \left\langle A \wedge_{i}(\operatorname{bkgd} e i), A \wedge_{i}(\text { base } e i)\right\rangle \\
\rangle &
\end{aligned}
$$


- Copy the left context: $\gamma_{i} \rightarrow s \rightarrow s \rightarrow \gamma_{i}$

$$
\begin{array}{r}
\text { copy_context } \triangleq \lambda e i j \cdot\langle(\text { woi } e), \\
\text { up_genv }
\end{array}
$$

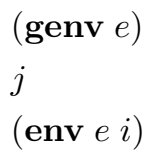

$($ env $e i)$

- Reset the base in a left context: $\gamma_{i} \rightarrow s \rightarrow \gamma_{i}$

$$
\begin{aligned}
\text { reset_base } \triangleq \lambda e i . & \\
\qquad \text { up_genv } & \\
& (\text { genv } e) \\
& i \\
& \left\langle(\mathbf{b k g d} e i), \top_{i}\right\rangle
\end{aligned}
$$

- The empty left context: $\gamma_{i}$

$$
\mathrm{nil}_{i} \triangleq\left\langle\mathbf{H}, \lambda i .\left\langle\top_{i}, \top_{i}\right\rangle\right\rangle
$$

- The empty right context: $\gamma_{i} \rightarrow o_{i}$

$$
\operatorname{stop}_{i} \triangleq \lambda e . \top_{i}
$$

\subsection{Dynamic Translation}

In this subsection, we continue with the formal details of M-TTDL, focusing on the dynamic logic and the systematic dynamic translation.

First of all, as we explained before, M-TTDL parallels TTDL in the aspect of the way to interpret sentences and discourses: both of them are functions from left contexts to right contexts to propositions. By contrasting formulas 2.2, 2.3 with 4.4, 4.5, we see that in TTDL, its type is $\gamma \rightarrow(\gamma \rightarrow o) \rightarrow o$, while in M-TTDL, it is $\gamma_{i} \rightarrow\left(\gamma_{i} \rightarrow o_{i}\right) \rightarrow o_{i}$ (the types of the latter are indexed with $i$ because the notion of possible world is incorporated). Because of that, as the default connective between sentences in a discourse, the dynamic conjunction in M-TTDL is defined exactly the same as in TTDL:

$$
\wedge_{M-T T D L}^{d} \triangleq \lambda A B e \phi . A e\left(\lambda e^{\prime} \cdot B e^{\prime} \phi\right)
$$

In order to negate a dynamic proposition in M-TTDL, we propose the following negation operator:

$$
\neg_{M-T T D L}^{d} \triangleq \lambda A e \phi \cdot \neg_{i}\left(A e \mathbf{s t o p}_{i}\right) \wedge_{i} \phi e
$$


When a proposition is negated, its context change potential will be restrained. This explains the modalized empty continuation $\mathbf{s t o p}_{i}$ in the above definition. It prevents the information in the left context to be updated in future discourse. Contrasting $\neg_{M-T T D L}^{d}$ (formula 4.25 with $\neg_{T T D L}^{d}$ (formula 2.6), we see that the two operators are in a completely similar structure, except for that the logical constants in $\neg_{T T D L}^{d}$ (i.e., $\neg, \top$ and $\wedge$ ) are substituted by their modal counterparts in $\neg_{M-T T D L}^{d}$ (i.e., $\neg_{i}, \top_{i}$ and $\wedge_{i}$ ).

For the dynamic existential quantifier (the one which ranges over individual variables) in M-TTDL, we propose the following definition:

$$
{ }^{\iota} \exists_{M-T T D L}^{d} \triangleq \lambda \operatorname{Pe\phi .}\left({ }^{\iota} \exists_{i}(\lambda x . P x e \phi)\right)
$$

Compared with its predecessor $\exists_{T T D L}^{d}$ (formula 2.7), the job of ${ }^{\iota} \exists_{M-T T D L}^{d}$ is less crucial. The quantifier ${ }^{\iota} \exists_{M-T T D L}^{d}$ does not update variables to the left context, because the structure of the left context is totally changed. In M-TTDL, the left context consists of propositions rather than invididuals. For more discussion, please refer back to section 4.1

Based on the above analysis, we will now present the systematic dynamic translation in M-TTDL. To distinguish the translations in M-TTDL from the previous one in TTDL, we introduce the $m$-bar notation.

Notation 4.1. We use the $m$-bar notation, for instance, $\bar{\tau}^{\mathrm{m}}$ or $\bar{M}^{\mathrm{m}}$, to denote the dynamic translation of a type $\tau$ or a $\lambda$-term $M$ in M-TTDL.

The dynamic translation of types in M-TTDL is in a parallel structure with the ones in TTDL. One may compare the following definition with definitions 2.2 .

Definition 4.3. The dynamic translation of a type $\tau \in T: \bar{\tau}^{\mathrm{m}}$, is defined inductively as follows:

1. $\bar{\iota}^{\mathrm{m}}=\iota$

2. ${\overline{o_{i}}}^{\mathrm{m}}=\Omega_{i}$;

3. $\overline{\sigma \rightarrow} \bar{\tau}^{\mathrm{m}}=\bar{\sigma}^{\mathrm{m}} \rightarrow \bar{\tau}^{\mathrm{m}}$, where $\tau, \sigma \in T$.

The detailed unfolding of $\Omega_{i}$ can be found in formula 4.7. Again, for the dynamic translation of $\lambda$-terms, we need to define the two functions: $\mathbb{D}^{m}$ and $\mathbb{S}^{m}$, which will be used to translate non-logical constants. These two function in M-TTDL are slightly different from their previous versions in TTDL (definition 2.3.

Definition 4.4. The dynamization function $\mathbb{D}_{\tau}^{m}$, which takes an input $\lambda$-term $A$ of type $\left(\gamma_{i} \rightarrow \tau\right)$, returns an output $\lambda$-term $A^{\prime}$ of type 
$\bar{\tau}^{\mathrm{m}}$; the staticization function $\mathbb{S}_{\tau}^{m}$, which takes an input $\lambda$-term $A^{\prime}$ of type $\bar{\tau}^{\mathrm{m}}$, returns an output $\lambda$-term $A$ of type $\gamma_{i} \rightarrow \tau$. In the following formulas, $e$ denotes a variable of type $\gamma_{i}$.

- $\mathbb{D}_{\tau}^{m}$ is defined inductively on type $\tau$ as follows:
$1 . \mathbb{D}_{\iota}^{m} A=A$ nil $_{i}$;
$2 . \mathbb{D}_{o_{i}}^{m} A=\lambda e \phi i .($ Aei $\wedge \phi($ up_context $e i(A e)) i)$;
$3 . \mathbb{D}_{\alpha \rightarrow \beta}^{m} A=\lambda x \cdot \mathbb{D}_{\beta}^{m}\left(\lambda e . A e\left(\mathbb{S}_{\alpha}^{m} x e\right)\right)$.

- $\mathbb{S}_{\tau}^{m}$ is defined inductively on type $\tau$ as follows:

$$
\begin{aligned}
& 1 . \mathbb{S}_{\iota}^{m} A^{\prime}=\lambda e \cdot A^{\prime} \\
& 2 . \mathbb{S}_{o_{i}}^{m} A^{\prime}=\lambda e \cdot A^{\prime} \text { e } \mathbf{s t o p}_{i} ; \\
& 3 . \mathbb{S}_{\alpha \rightarrow \beta}^{m} A^{\prime}=\lambda e \cdot\left(\lambda x . \mathbb{S}_{\beta}^{m}\left(A^{\prime}\left(\mathbb{D}_{\alpha}^{m}\left(\lambda e^{\prime} . x\right)\right)\right) e\right) .
\end{aligned}
$$

In the previous framework TTDL, the change of context is achieved through the dynamic existential quantifier (formulas 2.7). However, since the left context is interpreted differently in M-TTDL, the function $D_{o_{i}}^{m}$ is designed in a way such that it changes the current left context by inserting the dynamized modal proposition into the environment. For more discussions on the general cases of $\mathbb{D}^{m}$ and $\mathbb{S}^{m}$, please refer back to section 2. Below, we present the dynamic translation of $\lambda$-terms in M-TTDL, which is similar to that in TTDL as well. Compare the following definition with definitions 2.4 .

Definition 4.5. The dynamic translation of a $\lambda$-term $M$ (of type $\tau): \bar{M}^{\mathrm{m}}$, which is another $\lambda$-term of type $\bar{\tau}^{\mathrm{m}}$, is defined as follows:

1. $\bar{x}^{\mathrm{m}}=x$, if $x \in \mathcal{X}$;

2. $\overline{\mathbf{a}}^{\mathrm{m}}=\mathbb{D}_{\tau}^{m}(\lambda e \cdot \mathbf{a})$, if $\mathbf{a} \in \mathcal{C}_{N L}$;

3. $\bar{\wedge}^{\mathrm{m}}=\wedge_{M-T T D L}^{d}$, see formula 4.24 .

4. $\bar{\neg}^{\mathrm{m}}=\neg_{M-T T D L}^{d}$, see formula 4.25 .

5. $\bar{\exists}^{\mathrm{m}}={ }^{\iota} \exists_{M-T T D L}^{d}$, see formula 4.26 ,

6. $\overline{(M N)}^{\mathrm{m}}=\left(\bar{M}^{\mathrm{m}} \bar{N}^{\mathrm{m}}\right)$;

7. $\overline{(\lambda x \cdot M)}^{\mathrm{m}}=\left(\lambda x \cdot \bar{M}^{\mathrm{m}}\right)$.

For the dynamic translation of other logical constants such as $V$ (disjunction), $\rightarrow$ (implication), and $\forall$ (universal quantifier), we can apply the corresponding rules in definition 4.5 to their derived terms. Take implication for instance:

$$
\begin{aligned}
\overline{A \rightarrow B}^{\mathrm{m}} & =\overline{\neg(A \wedge \neg B)}^{\mathrm{m}} \\
& =\bar{\neg}^{\mathrm{m}}\left(\bar{A}^{\mathrm{m}} \bar{\wedge}^{\mathrm{m}}\left(\bar{\neg}^{\mathrm{m}} \bar{B}^{\mathrm{m}}\right)\right) \\
& =\neg_{M-T T D L}^{d}\left(\bar{A}^{\mathrm{m}} \wedge_{M-T T D L}^{d}\left(\neg_{M-T T D L}^{d} \bar{B}^{\mathrm{m}}\right)\right) \\
& \rightarrow_{\beta} \lambda e \phi . \neg_{i}\left(\bar{A}^{\mathrm{m}} e\left(\lambda e^{\prime} \cdot \neg_{i}\left(\bar{B}^{\mathrm{m}} e^{\prime} \mathbf{s t o p}_{i}\right)\right)\right) \wedge_{i} \phi e
\end{aligned}
$$


As to the semantics of M-TTDL, it follows from TTDL, which is also the same as in FOL, we shall not discuss that any further. The rest of this section is organized as follows. In the next subsection, we will provide the specific lexical entries around modality, which are mainly established based on the functions introduced in section 4.2. Then in section 4.5. we will focus on the relation between M-TTDL and TTDL: they are proved to have the same empirical predictions when no modality is concerned. Finally, applications of M-TTDL will be illustrated with specific linguistic examples in section 4.6 .

\subsection{Lexical Entries for Modals}

Based on the above analysis, in particular the fundamental functions in section 4.2, we propose the core of M-TTDL in this subsection, namely the specific lexical entries for modal expressions. We will first present the logical representations of the two modal operators: $\diamond$ and $\square$, which express possible modality and necessary modality, respectively; then we will introduce the function at, which explicitly indicates the world at which a dynamic proposition is to be evaluated; finally, two semantic entries corresponding to the epistemic modals in natural language: might and would, will be established based on the preceding knowledge. To save space, we shall only present the definition of each entry, more details can be found in section 2 of the appendix.

\section{Possibility Modal Operator}

The modal operator $\diamond$ takes a dynamic proposition $A$ (of type $\Omega_{i}$ ) as input, and returns another dynamic proposition $\diamond A$, which contains an existential modality. Hence the operator $\diamond$ should be of type $\Omega_{i} \rightarrow \Omega_{i}$. Its entry is defined as follows:

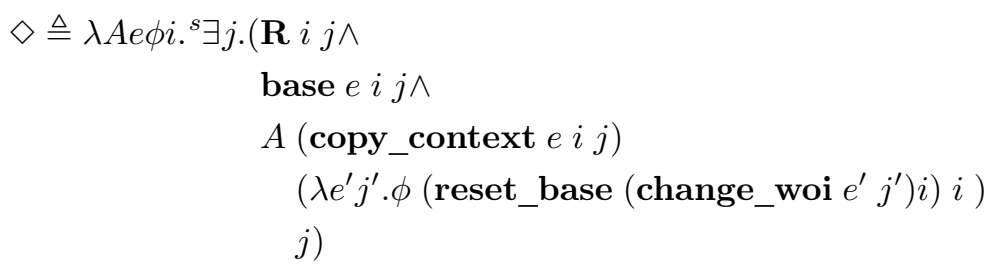

\section{Necessity Modal Operator}

The modal operator $\square$ is the one which creates a modality of universal force. It takes a dynamic proposition, $A$ (of type $\Omega_{i}$ ) for instance, as input, and returns a modalized proposition $\square A$. Hence same as $\diamond$, the operator $\square$ should also be of type $\Omega_{i} \rightarrow \Omega_{i}$. The entry is defined as 
follows:

$\square \triangleq \lambda \operatorname{Ae\phi i} \cdot\left({ }^{s} \forall j \cdot(\mathbf{R} i j \rightarrow\right.$

$$
\begin{aligned}
& (\text { base } e i j \rightarrow \\
& \left.\left.\left.\qquad\left(A(\text { copy_context } e i j) \text { stop }_{i} j\right)\right)\right)\right)
\end{aligned}
$$

$\wedge \phi e i$

\section{Evaluation "at" Some Possible World}

A big difference between the interpretation of propositions in classical logic and modal logic is that, a proposition is evaluated at a specific possible world in the latter system. Below, we will introduce the function at, which aims to associate a (modal) proposition with a possible world.

Intuitively, the at function picks up a particular world for a proposition, where it is to be evaluated. Hence its semantic type ought to be: $s \rightarrow \Omega_{i} \rightarrow \Omega_{i}$, where $s$ denotes the target world, the first $\Omega_{i}$ denotes the input proposition, and the second $\Omega_{i}$ is the output proposition with the world information interpolated. We propose its detailed semantic entry as follows:

$$
\begin{aligned}
& \text { at } \triangleq \lambda j A e \phi i . \\
& \text { if }(j=i) \\
& (A e \phi i) \\
& (\text { base } e i j \wedge \\
& A(\text { up_context } e j(\text { base } e i)) \\
& \left(\lambda e^{\prime} j^{\prime} . \phi\left(\text { reset_base } e^{\prime} i\right) i\right) \\
& j)
\end{aligned}
$$

\section{Modal Expressions}

In this subsection, we propose the semantic entries for the linguistic expressions which trigger epistemic modality, namely the modals: might and would. The technical details will largely depend on what we have introduced above. Basically, we will show how to build up complex entries with $\diamond, \square$, and at. We shall start with might, then address would.

The modal verb might, which introduces the epistemic possibility modality, is of type $\Omega_{i} \rightarrow \Omega_{i}$. We propose the lexical entry of might as follows:

$$
\llbracket m i g h t \rrbracket_{M-T T D L}=\lambda A e \phi i .(\text { at }(\text { woi } e)(\diamond A)) e \phi i
$$

The intuition behind the above formula is as follows: when we say something might happen, it means that at a particular world (the 
salient world), the proposition is possibly true, which logically denotes that the proposition is satisfied at some accessible world. This is exactly the meaning born within the entry of might as in formula 4.31 a dynamic proposition $A$ is possibly true with regard to the salient world.

Same as might, the entry for the modal verb would is also of type $\Omega_{i} \rightarrow \Omega_{i}$. Its representation is also made up of previously introduced entries:

$$
\llbracket \text { would } \rrbracket_{M-T T D L}=\lambda A e \phi i .(\text { at }(\text { woi } e)(\square A)) e \phi i
$$

The intuition behind the above formula is as follows: when we say something would happen, it means at the salient world, the proposition is necessarily true, which logically denotes that the proposition is satisfied at every accessible world. This is exactly the meaning born within the entry of would as in formula 4.32 a dynamic proposition $A$ is necessarily true with regard to the salient world.

\subsection{From TTDL to M-TTDL}

With the above definition of the framework M-TTDL, we would like to further examine the formal relation between TTDL and M-TTDL. Namely, if M-TTDL is an extension of TTDL, it should be able to cover the paradigm phenomena that dynamic semantics systems are designed to solve. Again, to save space, we will only sketch the proof, rather than presenting the full-blown rigorous exposition.

Since formulas in TTDL and M-TTDL have very different forms, it is difficult to compare the two systems in a straightforward way. Hence we first need to introduce a bridging framework, which is syntactically similar to TTDL. We call the new framework Propositional TTDL (P-TTDL), because the left context in it is updated with propositions, instead of discourse referent:7. This is exactly the case in M-TTDL, see formula 4.3. More specifically, P-TTDL is defined upon M-TTDL by getting rid of modality. In other words, P-TTDL is a simplified variant of M-TTDL such that it is not concerned with possible worlds.

The next step is to show that the two frameworks: P-TTDL and TTDL, will always obtain the same result in handling discourse semantics. We found out that their results will only differ if the input formula contains free variables. However, this is not a concern because we are only interested in closed formulas 8 . Moreover, since P-TTDL is the unmodalized version of M-TTDL by definition, we may thus con-

\footnotetext{
${ }^{7}$ P-TTDL is very similar to the framework GL presented in (Lebedeva 2012).

${ }^{8}$ Here we refer to logical formulas under dynamic frameworks such as T"TDL. For instance, pronominal anaphora across sentence boundaries such as the one in "A man walks in the park. He whistles." will not introduce any free variable.
} 
clude that M-TTDL and TTDL are compatible in all cases where no modality is involved.

\subsection{Illustration}

\section{Other Lexical Entries}

With the systematic way of dynamization in section 4.3 , we can obtain the semantic representation for other linguistic elements in a purely compositional way. We will conduct the step by step translation for two lexical entries. First, we look at the common noun wolf.

1. The standard entry for wolf:

$$
\llbracket w o l f \rrbracket=\lambda x . \text { wolf } x
$$

It takes an individual as input, and yields a proposition, its type is $\iota \rightarrow o$.

2. According to definition 4.5 .

$$
\overline{\llbracket w o l f \rrbracket}^{\mathrm{m}}=\overline{\lambda x \text {.wolf } x}^{\mathrm{m}}=\lambda x \text {. } \overline{\text { wolf } x}^{\mathrm{m}}=\lambda x \cdot \overline{\text { wolf }}^{\mathrm{m}} x
$$

3. The predicate constant wolf is of type $\iota \rightarrow o$, hence according to definition 4.4 .

$$
\begin{aligned}
\overline{\text { wolf }}^{m} & =\mathbb{D}_{\iota \rightarrow o_{i}}^{m}(\lambda e \cdot \text { wolf }) \\
& =\lambda x \cdot \mathbb{D}_{o_{i}}^{m}\left(\lambda e \cdot\left(\lambda e^{\prime} \cdot \mathbf{w o l f}\right) e\left(\mathbb{S}_{\iota}^{d n} x e\right)\right) \\
& \rightarrow \beta \lambda x \cdot \mathbb{D}_{o_{i}}^{m}\left(\lambda e \cdot \mathbf{w o l f}\left(\mathbb{S}_{\iota}^{d n} x e\right)\right) \\
& =\lambda x \cdot \mathbb{D}_{o_{i}}^{m}(\lambda e \cdot \text { wolf } x) \\
& =\lambda x e \phi i .\left(\left(\lambda e^{\prime} \text {.wolf } x\right) e i \wedge \phi\left(\mathbf{u p} \_ \text {context } e i\left(\left(\lambda e^{\prime} \cdot \text { wolf } x\right) e\right)\right) i\right) \\
& \rightarrow \beta \lambda x e \phi i .(\text { wolf } x i \wedge \phi(\mathbf{u p} \text { _context } e i(\text { wolf } x)) i)
\end{aligned}
$$

4. As a result, based on the result of the previous step, we can obtain:

$$
\begin{aligned}
\overline{\llbracket w o l f \rrbracket}^{\mathrm{m}} & =\lambda x \cdot \overline{\text { wolf }}^{\mathrm{m}} x \\
& =\lambda x \cdot\left(\lambda x^{\prime} e \phi i .\left(\text { wolf } x^{\prime} i \wedge \phi\left(\mathbf{u p \_ c o n t e x t} e i\left(\text { wolf } x^{\prime}\right)\right) i\right)\right) x \\
& \rightarrow \beta \lambda x e \phi i .(\text { wolf } x i \wedge \phi(\text { up_context } e i(\text { wolf } x)) i)
\end{aligned}
$$

In the above formula, $x$ is of type $\iota$, hence $\overline{\llbracket w o l f \rrbracket}^{\mathrm{m}}$ is of type $\iota \rightarrow$ $\Omega_{i}$. Same as for previous entries involving elementary functions, we will not unfold the complete entry.

For the indefinite article $a$, its stepwise dynamization is as follows:

1. The standard entry for $a$ :

$$
\llbracket a \rrbracket=\lambda P Q \cdot \exists(\lambda x . P x \wedge Q x)
$$


It takes two properties and returns a proposition, its type is $(\iota \rightarrow$ $o) \rightarrow(\iota \rightarrow o) \rightarrow o$.

2. According to definition 4.5 .

$$
\begin{aligned}
\overline{\llbracket a \rrbracket}^{\mathrm{m}} & =\overline{\lambda P Q \cdot \exists(\lambda x \cdot P x \wedge Q x)}^{\mathrm{m}} \\
& =\lambda P Q \cdot \bar{\exists}^{\mathrm{m} x \cdot P x \wedge Q x)} \\
& =\lambda P Q \cdot \bar{\exists}^{\mathrm{m}}\left(\lambda x \cdot P x \bar{\wedge}^{\mathrm{m}} Q x\right) \\
& =\lambda P Q \cdot \cdot^{\iota} \exists_{M-T T D L}^{d}\left(\lambda x \cdot P x \wedge_{M-T T D L}^{d} Q x\right)
\end{aligned}
$$

3. According to formula 4.24 and 4.26

$$
\begin{aligned}
\overline{\llbracket a \rrbracket} \mathrm{m}= & \lambda P Q \cdot{ }^{\iota} \exists_{M-T T D L}^{d}\left(\lambda x \cdot P x \wedge_{M-T T D L}^{d} Q x\right) \\
= & \lambda P Q \cdot\left(\lambda P^{\prime} e \phi \cdot\left({ }^{\iota} \exists_{i}\left(\lambda x \cdot P^{\prime} x e \phi\right)\right)\right) \\
& \quad\left(\lambda x \cdot\left(\lambda A B e \phi \cdot A e\left(\lambda e^{\prime} \cdot B e^{\prime} \phi\right)\right)(P x)(Q x)\right) \\
\rightarrow & \lambda P Q e \phi{ }^{\iota} \exists_{i}\left(\lambda x \cdot P x e\left(\lambda e^{\prime} \cdot Q x e^{\prime} \phi\right)\right) \\
= & \lambda P Q e \phi i \cdot{ }^{\iota} \exists\left(\lambda x \cdot P x e\left(\lambda e^{\prime} \cdot Q x e^{\prime} \phi\right) i\right)
\end{aligned}
$$

$P$ and $Q$ are of type $\iota \rightarrow \Omega_{i}$, hence $\overline{\llbracket a \rrbracket}^{\mathrm{m}}$ is of type $\left(\iota \rightarrow \Omega_{i}\right) \rightarrow$ $\left(\iota \rightarrow \Omega_{i}\right) \rightarrow \Omega_{i}$.

The translations of other syntactic categories should be rather straightforward. Before closing this subsection, we would like to draw attention to the lexical entry of pronouns. Syntactically, a pronoun belongs to the NP category. Its semantic type ought to be $(\iota \rightarrow o) \rightarrow o$. However, in standard logical semantics such as MG, no explicit entry for pronoun is provided. It is simply treated as a variable bound by the quantifier from the antecedent in standard logical semantics. In the vocabulary of M-TTDL, we have introduced the choice operator sel. Different from the one in previous frameworks, the sel in M-TTDL is of type $(\iota \rightarrow o) \rightarrow o \rightarrow \iota$, based on an input property, it retrieves an individual from the background proposition. So the dynamic entry for pronoun, such as he and it, can be given as follows:

$$
\overline{\llbracket h e \rrbracket}^{\mathrm{m}}=\lambda P e \phi i . P(\operatorname{sel}(\lambda x \cdot \operatorname{human} x i \wedge \text { male } x i)(\text { bkgd } e i i)) e \phi i
$$

$$
\overline{\llbracket i t \rrbracket}^{\mathrm{m}}=\lambda P e \phi i . P(\operatorname{sel}(\lambda x . \neg(\text { human } x i))(\operatorname{bkgd} e i i)) e \phi i
$$

$P$ is of type $\iota \rightarrow \Omega_{i}$, hence both $\overline{\llbracket h e \rrbracket}^{\mathrm{m}}$ and $\overline{\llbracket i t \rrbracket}^{\mathrm{m}}$ are of type $(\iota \rightarrow$ $\left.\Omega_{i}\right) \rightarrow \Omega_{i}$.

\section{Discourses}

The purpose of this subsection is to show how the framework M-TTDL can be applied to handle linguistic examples, which are concerned with 
modality, in particular modal subordination. In what follows, we will compositionally compute the logical representation of the discourse based on the above proposed lexical entries.

Firstly, let's start with a simple example, where modality is only involved in the second part of the discourse, hence the indicated anaphoric link is felicitous.

$\underline{\mathrm{A} \mathrm{wolf}}_{i}$ walks in. $\underline{\mathrm{It}}_{i}$ might growl.

The first sentence in example (12) does not contain modality. Its semantic representation in M-TTDL can be computed as follows:

$$
\llbracket(12)-1 \rrbracket_{M-T T D L}=\overline{\llbracket w a l k \_i n \rrbracket(\llbracket a \rrbracket \llbracket w o l f \rrbracket)}^{\mathrm{m}}
$$

The second part, where the modal might appears, is translated in the following way:

$$
\llbracket(12) \cdot 2 \rrbracket_{M-T T D L}=\llbracket \text { might } \rrbracket_{M-T T D L} \overline{(\llbracket \text { growl } \rrbracket \llbracket i t \rrbracket)}^{\mathrm{m}}
$$

The detailed lexical entry for pronoun it can be found in formula 4.36. Notice that the choice operator sel in M-TTDL has a different type as the one in TTDL (see definition 4.1). We do not give the complete unfolding of the logical formulas because they are huge. Instead, we will directly present the result of discourse incrementation, which will be applied to the empty left context nil $_{i}$, the empty continuation $\mathbf{s t o p}_{i}$, and the world constant $\mathbf{H}$. As before, assume the conjunction is the connective for sentence sequencing, then:

\section{$\llbracket(12) \rrbracket_{M-T T D L} \operatorname{nil}_{i} \operatorname{stop}_{i} \mathbf{H}$}

$=\left(\llbracket(12)-1 \rrbracket_{M-T T D L} \bar{\Lambda}^{\mathrm{m}} \llbracket(12)-2 \rrbracket_{M-T T D L}\right) \operatorname{nil}_{i} \operatorname{stop}_{i} \mathbf{H}$

${ }^{\circ}{ }^{\iota} \exists x$. (wolf $x \mathbf{H} \wedge$ walk_in $x \mathbf{H} \wedge$

${ }^{s} \exists j .(\mathbf{R} \mathbf{H} j \wedge(($ walk_in $x j \wedge$ wolf $x j) \wedge$

growl $(\operatorname{sel}(\lambda x . \neg($ human $x j))($ walk_in $x j \wedge$ wolf $x j)) j)))$

In the above formula, the choice operator sel should select a variable from its second argument: the proposition (walk_in $x j \wedge$ wolf $x j$ ), based on the criteria from its first argument, namely $\lambda x . \neg($ human $x j)$. Since variable $x$ is the only candidate, assume sel makes the correct 
choice 9 the above representation can be further reduced into:

$\llbracket(12) \rrbracket_{M-T T D L} \mathrm{nil}_{i}$ stop $\mathbf{H}$

$\rightarrow \beta{ }^{\iota} \exists x$. (wolf $x \mathbf{H} \wedge$ walk_in $x \mathbf{H} \wedge$

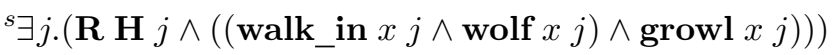

Assuming $\mathbf{H}$ is the world of utterance, the semantics of the above formula is: there is a wolf which walks in at the actual world $\mathbf{H}$, and at an accessible possible world $j$, there is also a wolf which walks in, and it also growls at $j$. This is exactly what (12) means.

In addition, the framework M-TTDL can successfully block the infelicitous anaphors as in the following examples, where the referents are introduced within the scope of modal operators:

a. $\quad \mathrm{A} \mathrm{wolf}_{i}$ might walk in. ${ }^{*} \mathrm{It}_{i}$ growls.

b. $\underline{\mathrm{A} \mathrm{wolf}}_{i}$ would walk in. ${ }^{*} \underline{\mathrm{It}}_{i}$ growls.

The interpretations of the first two sentences are calculated as follows:

$$
\begin{aligned}
& \llbracket(13-\mathrm{a})-1 \rrbracket_{M-T T D L}=\llbracket \text { might } \rrbracket_{M-T T D L} \overline{\left(\llbracket w a l k \_i n \rrbracket((\llbracket a \rrbracket \llbracket w o l f \rrbracket))\right.}^{\mathrm{m}} \\
& \llbracket(13-\mathrm{b})-1 \rrbracket_{M-T T D L}=\llbracket \text { would } \rrbracket_{M-T T D L} \overline{\left(\llbracket w a l k \_i n \rrbracket(\llbracket a \rrbracket \llbracket w o l f \rrbracket)\right)}^{\mathrm{m}}
\end{aligned}
$$

They share the same second part:

$$
\llbracket(13-\mathrm{a})-2 \rrbracket_{M-T T D L}=\llbracket(13-\mathrm{b})-2 \rrbracket_{M-T T D L}=\overline{\llbracket \text { growl } \rrbracket \llbracket i t \rrbracket}^{\mathrm{m}}
$$

The following steps are the same as for the previous example, we will give the final result directly. For (13-a).

$$
\begin{aligned}
& \text { 【(13-a) } \rrbracket_{M-T T D L} \text { nil }_{i} \mathbf{s t o p}_{i} \mathbf{H} \\
& =\left(\llbracket(13-\mathrm{a})-1 \rrbracket_{M-T T D L} \bar{\Lambda}^{\mathrm{m}} \llbracket(13-\mathrm{a})-2 \rrbracket_{M-T T D L}\right) \text { nil }_{i} \operatorname{stop}_{i} \mathbf{H} \\
& { }_{\beta}{ }^{s} \exists j \text {. (R H } j \wedge{ }^{\iota} \exists x \text {. (wolf } x j \wedge \text { (walk_in } x j \wedge \\
& \text { growl (sel }(\lambda x . \neg(\text { human } x \mathbf{H})) \top) \mathbf{H})))
\end{aligned}
$$

The above formula means that there is an accessible world $j$ from the actual world $\mathbf{H}$, in which a wolf walks in. And at the actual world, there is some individual that growls. But since the choice operator sel does not have a proper proposition from which it can pick up a referent, the anaphora in (13-a) cannot be resolved. Assume $A$ is the proposition expressed by a wolf walks in, $B$ is the one expressed by it

\footnotetext{
${ }^{9}$ Since we stick to the standard lexical entry of wolf (formula 4.33 , its dynamic entry (formula 4.34 does not indicate whether a wolf is human or not. We radically simplify this issue by assuming a powerful choice operator sel, which can resolve the satisfied variable by itself. However, an enriched lexical entry would be desirable in the future work.
} 
growls, $\mathbf{M}$ denotes the entry of might, then the possible worlds hierarchy of example (13-a) is depicted in figure 1

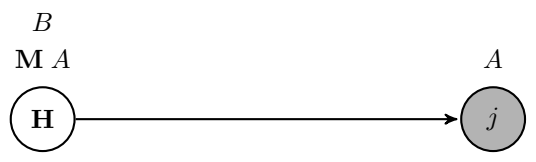

FIGURE 1 Possible Worlds Hierarchy of Example (13-a)

The anaphor it occurs at world $\mathbf{H}$, while the referent corresponding to a wolf is introduced in $j$. As a result, the anaphoric link cannot be resolved. It is the similar case for (13-b), although the detailed logical representation is different:

$$
\begin{aligned}
& \llbracket(13-\mathrm{b}) \rrbracket_{M-T T D L} \operatorname{nil}_{i} \mathbf{s t o p}_{i} \mathbf{H} \\
& =\llbracket(13-\mathrm{b})-1 \rrbracket_{M-T T D L} \bar{\wedge}^{\mathrm{m}} \llbracket(13-\mathrm{b})-2 \rrbracket_{M-T T D L} \text { nil }_{i} \mathbf{s t o p}_{i} \mathbf{H} \\
& { }_{\beta}{ }^{{ }^{s} \forall j .(\mathbf{R ~ H} j} \rightarrow{ }^{{ }} \exists x .(\text { wolf } x j \wedge(\text { walk_in } x j \wedge \\
& \quad \text { growl }(\operatorname{sel}(\lambda x . \neg(\text { human } x \mathbf{H})) \top) \mathbf{H})))
\end{aligned}
$$

The above formula means that for all accessible worlds from the actual world $\mathbf{H}$, there is a wolf walking in at it. At the same time, there is some individual who growls at the actual world. But this growing individual cannot be properly resolved as any referent. Its possible worlds hierarchy is provided in figure 2 .

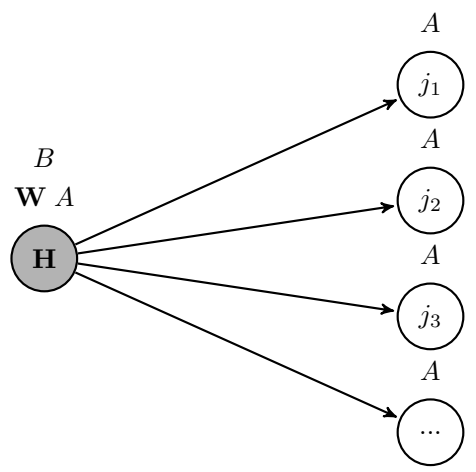

FIGURE 2 Possible Worlds Hierarchy of Example (13-b)

No discourse referent is introduced at world $\mathbf{H}$, where the pronoun 
it occurs. Hence the anaphor is problematic.

Now let's consider a more complex discourse concerning modal subordination, where modalities are involved in both component sentences. It is in a parallel structure as example (6) in section 1.2 .

$$
\frac{\text { A wolf }}{i 2011} \text { might walk in. } \underline{\text { It }}_{i} \text { would growl. Asher and Pogodalla }
$$

The first sentence of (14) is identical to (13-a) 1 , hence:

$$
\begin{aligned}
\llbracket(14)-1 \rrbracket_{M-T T D L} & =\llbracket(13-\mathrm{a})-1 \rrbracket_{M-T T D L} \\
& =\llbracket \text { might } \rrbracket_{M-T T D L} \overline{\left(\llbracket w a l k \_i n \rrbracket(\llbracket a \rrbracket \llbracket w o l f \rrbracket)\right)}
\end{aligned}
$$

The representation for the second sentence can be achieved in a similar way:

$$
\llbracket(14)-2 \rrbracket_{M-T T D L}=\llbracket \text { would } \rrbracket_{M-T T D L} \overline{(\llbracket \text { growl } \rrbracket \llbracket i t \rrbracket)}^{\mathrm{m}}
$$

Following the previous examples, the discourse incrementation for (14) is also straightforward. We will directly give the final result:

【(14) $M-T T D L \operatorname{nil}_{i} \operatorname{stop}_{i} \mathbf{H}$

$=\llbracket(14)-1 \rrbracket_{M-T T D L} \bar{\wedge}^{\mathrm{m}} \llbracket(14)-2 \rrbracket_{M-T T D L}$ nil $_{i} \mathbf{s t o p}_{i} \mathbf{H}$

$\rightarrow_{\beta}{ }^{s} \exists j .\left(\mathbf{R ~ H} j \wedge{ }^{\iota} \exists x\right.$. (wolf $x j \wedge$ walk_in $x j \wedge$

${ }^{s} \forall k .(\mathbf{R} j k \rightarrow(($ wolf $x k \wedge$ walk_in $x k) \rightarrow$

$($ growl $(\operatorname{sel}(\lambda x . \neg($ human $x k))($ wolf $x k \wedge$ walk_in $x k)) k))))$

Now the choice operator sel will select a non-human variable at world $k$ from the proposition (wolf $x k \wedge$ walk_in $x k$ ), where $x$ is the only possibility. Hence we can further reduce the above formula as follows: 【(14) $\rrbracket_{M-T T D L} \operatorname{nil}_{i} \mathbf{s t o p}_{i} \mathbf{H}$

${ }_{\beta}{ }^{s} \exists j$. (R H $j \wedge{ }^{\iota} \exists x$. (wolf $x j \wedge$ walk_in $x j \wedge$

$$
\left.\left.{ }^{s} \forall k .(\mathbf{R} j k \rightarrow((\text { wolf } x k \wedge \text { walk_in } x k) \rightarrow(\text { growl } x k)))\right)\right)
$$

This means there exists a possible world $j$ which is accessible from the actual world $\mathbf{H}$, a wolf walks in at $j$; and at every accessible world $k$ from $j$, if the wolf walks in, then it growls. This corresponds to the semantics of the original discourse (14). Again, we provide its possible worlds hierarchy as follows.

Finally, we will examine a last example, which switches back and forth between the modal mode and the factual mode. For the sake of convenience, we stick to the same vocabulary by simplifying example (18) from (Stone, 1999):

(15) $\quad \underline{\mathrm{A}}$ wolf $_{i}$ might walk in. John has $\underline{\operatorname{agun}}_{j}$. John would use it $\underline{\mathrm{it}}_{j}$ to

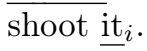




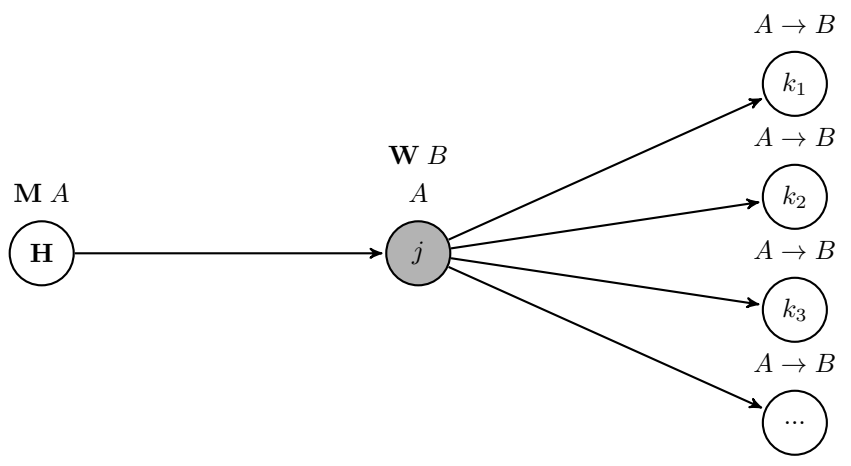

FIGURE 3 Possible Worlds Hierarchy of Example (14)

We first have to compute the semantic representation for each component sentence. As we can see, (15) 1 is exactly the same as (13-a) 1 and $(14)-1$, we will not repeat it here any more. For the remaining two sentences, we have:

$$
\llbracket(15)-2 \rrbracket_{M-T T D L}=\overline{\llbracket h a v e \rrbracket(\llbracket a \rrbracket \llbracket g u n \rrbracket) \llbracket J o h n \rrbracket}^{\mathrm{m}}
$$

$$
\llbracket(15)-3 \rrbracket_{M-T T D L}=\llbracket \text { would } \rrbracket_{M-T T D L} \overline{(\llbracket u s e \rrbracket \llbracket i t \rrbracket(\llbracket \operatorname{shoot} \rrbracket \llbracket i t \rrbracket) \llbracket J o h n \rrbracket)}^{\mathrm{m}}
$$

The semantic representation of the whole discourse is obtained by straightforwardly sequencing the three component sentences with dynamic conjunction. As before, we apply it to the empty left context nil $_{i}$, the empty continuation $\mathbf{s t o p}_{i}$, and the world constant $\mathbf{H}$. The reduced formula is as follows: 


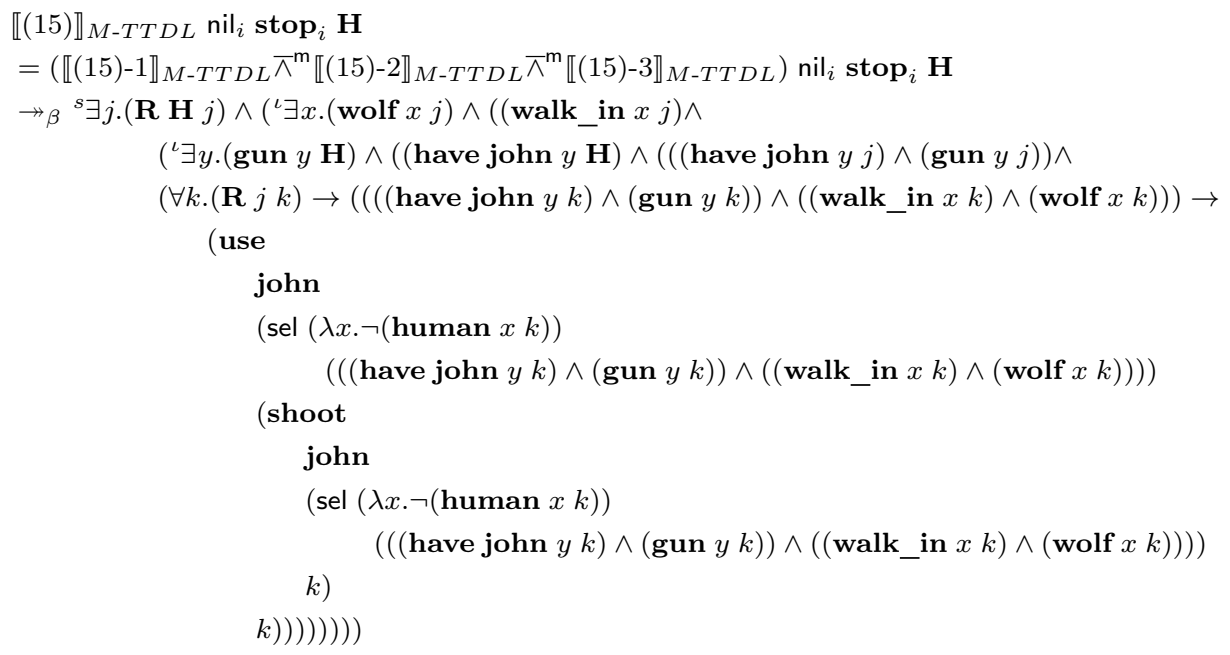

As we can see, both choice operators can select a non-human variable at world $k$ from the sub-formula $((($ have john $y k) \wedge($ gun $y k)) \wedge$ $(($ walk_in $x k) \wedge($ wolf $x k))$. Let's assume the first sel picks up $y$, the second picks up $x$, then the above formula can be further reduced to:

【(15) $\rrbracket_{M-T T D L}$ nil $_{i} \mathbf{s t o p}_{i} \mathbf{H}$

$$
\begin{aligned}
\rightarrow_{\beta}{ }^{s} \exists j .(\mathbf{R ~ H} j) & \wedge\left({ }^{\iota} \exists x .(\text { wolf } x j)\right. \\
\left({ }^{\iota} \exists y \cdot(\text { gun } y \mathbf{H}) \wedge((\text { have john } y \mathbf{H})\right. & \wedge(((\text { have john } y j) \wedge(\text { gun } y j)) \wedge \\
\left({ }^{s} \forall k .(\mathbf{R} j k) \rightarrow(((\text { have john } y k)\right. & \wedge(\text { gun } y k)) \wedge \\
((\text { walk_in } x k) \wedge(\text { wolf } x k))) & \rightarrow(\text { use john } y(\text { shoot john } x k) k))))))))
\end{aligned}
$$

The semantics of the above complex formula is: there is a possible world $j$ accessible from the actual world $\mathbf{H}$, a wolf walks in at $j$; furthermore, John owns a gun at the actual world $\mathbf{H}$; in addition, in every possible world $k$ which is accessible from $j$, if the wolf walks in, then John uses the gun to shoot the wolf. As a result, all the anaphoric links in discourse (15), which are across the modal mode and the factual mode, can be correctly accounted for.

\section{Conclusion and Future Work}

Anaphora interpretation is a critical piece of machinery in natural language interpretation. The aim of this paper was to study the semantics of one specific type of anaphora: inter-sentential pronominal anaphora from the discourse perspective. More specifically, this paper was concerned with the phenomenon of modal subordination, and the con- 
straints on interpretation of anaphors and modal sentences.

To summarize, the solution we proposed involves extending the dynamic framework TTDL. Another system in the simply typed $\lambda$ calculus family, namely M-TTDL, has been developed. We have briefly sketched the relation between M-TTDL and TTDL: the former has at least the same empirical coverage as the latter (the formal proof is not presented due to the length of the paper). Furthermore, M-TTDL is able to account for both the felicitous and infelicitous anaphors under various number of modality-involved discourses. TTDL and MTTDL have advantage over other dynamic theories because they stick to the traditional Montagovian style: the only operations involved are standard $\alpha$-conversions and $\beta$-reductions. Hence the principle of compositionality is retained without adding any new concept to classical logic.

However, the empirical data addressed here is only a small part in the semantics of anaphora and modality. On the theoretical side, a potential continuation of the current research is to broaden the coverage of our framework. For instance, in the following set of examples, the lifespan of a discourse referent is longer than M-TTDL would expect:

a. You must write a letter $i$ to your parents. $\underline{I t}_{i}$ has to be sent by airmail. The letter must get there by tomorrow. (Karttunen, 1969)

b. Mary wants to marry a rich $\operatorname{man}_{i}$. $\underline{H e}_{i}$ must be a banker. (Karttunen, 1969)

c. Harvey courts $\underline{\mathrm{a} \mathrm{girl}}_{i}$ at every convention. $\underline{\mathrm{She}}_{i}$ always comes to the banquet with him. The girl $i$ is usually very pretty. (Karttunen, 1969)

d. A train ${ }_{i}$ leaves every hour for Boston. It ${ }_{i}$ always stops in New Haven. (Sells, 1985)

e. Every chess set comes with a spare pawn ${ }_{i}$. $\underline{\mathrm{It}}_{i}$ is taped to the top of the box. (Sells, 1985)

At a first glance, the above examples, in particular, (16-c) (16-d) and (16-e), where no modality is involved, can be generalized as modal subordination. The quantifications in these examples can be treated in an analogous way as modality ${ }^{10}$, the quantification is over objects such as situation or time, while modality ranges over possible worlds. According to M-TTDL, the indefinite introduced under the scope of some modal operator is accessible to subsequent modal context. However,

\footnotetext{
${ }^{10}$ The second sentence in example (16-e) is assumed to contain a covert universal quantifier: it can be paraphrased as it is always taped to the top of the box.
} 
this rule is only admitted when the first modality is existential, which is not the case in any of the examples of $(16)$. To account for examples such as (16), one will have to investigate the environments under which the scope of universal modality can be extended. Obviously, the current version of M-TTDL does not take that into consideration.

In addition, the accessibility of discourse referents seems to be exceptional when modality and negation are involved at the same time:

John won't buy a car $i$ because he wouldn't have room for it $_{i}$ in his garage. (Partee, 1973)

In the above example, the first part is in factual mood, the second part is modalized, this pattern falls well under the cases to be handled in M-TTDL. However according to M-TTDL, discourse referents introduced in the scope of (single) negation is not accessible from outside. So the indefinite a car in (17) should not serve as antecedent for any subsequent anaphor. Nevertheless, the anaphoric link in (17) is fairly acceptable. As suggested by (Partee, 1973), the auxiliary would requires the presence of a subordinate clause with if or unless, except when it is used to express volition or habit. Hence (17) can be regarded as an abbreviation for the following sentence:

(18) John won't buy a car because if he did buy a car $i$, he wouldn't have room for $\underline{i t}_{i}$ in his garage. (Partee, 1973)

In the paraphrase (18) there are two occurrences of $a$ car. The pronoun it is anaphorically related to the second occurrence rather than the first one. With this paraphrase, the anaphor can be successfully accounted for in M-TTDL. Similar examples include:

(19) a. I didn't submit a paper ${ }_{i}$. They wouldn't have published it $_{i}$. Kibble, 1994)

b. John didn't buy a mystery novel ${ }_{i}$. He would be reading $\underline{i t}_{i}$ by now. (Krifka, 2001)

c. Mary didn't buy a microwave $i$. She would never use $\underline{\text { it }}_{i}$. (Frank, 1997)

d. Fred didn't draw a picture ${ }_{i}$. He would have made a mess of it $_{i}$. Frank, 1997)

As in example (18), the discourses in (19) can be paraphrased as follows:

(20) a. I didn't submit a paper. If I had submitted a paper ${ }_{i}$, they wouldn't have published it $_{i}$. Kibble, 1994) 
b. John didn't buy a mystery novel. If he had bought a mystery novel $_{i}$, he would be reading $\underline{i t}_{i}$ by now. (Krifka). 2001)

c. Mary didn't buy a microwave because if she had bought a $\underline{\text { microwave }}_{i}$ she would never have used $\underline{i t}_{i}$. (Frank, 1997)

d. Fred didn't draw a picture because if he had drawn a $\underline{\text { picture }}_{i}$ he would have made a mess of $\underline{i t}_{i}$. (Frank, 1997)

The interesting thing we can draw from the above examples is that, it is the counterfactual that has been accommodated in the modal base. To acquire M-TTDL with the ability to account for examples such as $(17)$ and (19), we should at least tackle two fundamental questions. Firstly, the condition that triggers the accommodation ought to be precisely specified, e.g., the presence of the modal auxiliary would. More importantly, we will need to determine which factual proposition(s) should be negated. It seems that we always accommodate the counterfactual of the nearest preceding sentence, but this generalization has to be verified by more examples.

Besides the above theoretical continuations, the current framework face a number of practical challenges as well. It is well-known that defining propositions as functions from possible worlds to truth values (this is what most modal systems do to integrate the notion of possible world) creates hyperintensional problems. Since M-TTDL is configured exactly in this fashion, it inevitably suffers from the same problems. Also, from the implementational point of view, possible worlds are costly and have been shown to present serious complexity problems even for the propositional case (Lappin, 2014). Consequently, it might not be an easy task to put the M-TTDL framework into practice in the short term, particularly in those real-time anaphora resolution systems. However, we are still full of optimism because a fragment of M-TTDL has already been successfully implemented in the Abstract Categorial Grammar toolkit (de Groote, 2001), programmed in the OCaml language. This can be considered as a first step towards a real application in language technologies, computational issues such as complexity will definitely be a direction for further research. 


\section{References}

Asher, Nicholas and Sylvain Pogodalla. 2011. A montagovian treatment of modal subordination. In 20th Semantics and Linguistic Theory conferenceSALT2010.

Church, Alonzo. 1940. A formulation of the simple theory of types. The journal of symbolic logic 5(2):56-68.

de Groote, Philippe. 2001. Towards abstract categorial grammars. In Proceedings of the 39th Annual Meeting on Association for Computational Linguistics, pages 252-259. Association for Computational Linguistics.

de Groote, Philippe. 2006. Towards a montagovian account of dynamics. In Proceedings of SALT, vol. 16, pages 1-16.

Frank, Anette. 1997. Context dependence in modal constructions. Ph.D. thesis, Universität Stuttgart.

Groenendijk, Jeroen and Martin Stokhof. 1991. Dynamic predicate logic. Linguistics and philosophy 14(1):39-100.

Heim, Irene. 1982. The semantics of definite and indefinite noun phrases. Ph.D. thesis, University of Massachusetts Amherst.

Heim, Irene. 1983. File change semantics and the familiarity theory of definiteness. Formal Semantics pages 223-248.

Kamp, Hans. 1981. A theory of truth and semantic representation. Formal Semantics pages 189-222.

Karttunen, Lauri. 1969. Discourse referents. In Proceedings of the 1969 conference on Computational linguistics, pages 1-38. Association for Computational Linguistics.

Kibble, Rodger. 1994. Dynamics of epistemic modality and anaphora. In International workshop on computational semantics, pages 121-130. Citeseer.

Kratzer, Angelika. 1977. What 'must' and 'can' must and can mean. Linguistics and Philosophy 1(3):337-355.

Kratzer, Angelika. 1981. The notional category of modality. Words, worlds, and contexts pages $38-74$.

Kratzer, Angelika. 1986. Conditionals. In Chicago Linguistics Society, vol. 22, pages $1-15$.

Kratzer, Angelika. 1991. Modality. Semantics: An international handbook of contemporary research pages 639-650.

Krifka, Manfred. 2001. Diskursrepräsentation und dynamische interpretation. University Lecture. Humboldt-Universität zu Berlin.

Kripke, Saul Aaron. 1959. A completeness theorem in modal logic. Journal of Symbolic Logic pages 1-14.

Kripke, Saul Aaron. 1963. Semantical analysis of modal logic i normal modal propositional calculi. Mathematical Logic Quarterly 9(5-6):67-96.

Lappin, Shalom. 2014. Curry typing, polymorphism, and fine-grained intensionality. In S. Lappin and C. Fox, eds., The Handbook of Contemporary Semantic Theory: Second Edition, chap. 13. Wiley-Blackwell. 
Lebedeva, Ekaterina. 2012. Expression de la dynamique du discours à l'aide de continuations. Ph.D. thesis, Université de Lorraine.

Montague, Richard. 1970a. English as a formal language. Linguaggi nella societae nella tecnica pages 189-224.

Montague, Richard. 1970b. Universal grammar. Theoria 36(3):373-398.

Montague, Richard. 1973. The proper treatment of quantification in ordinary english. In Approaches to Natural Language, vol. 49, pages 221-242. Dordrecht.

Partee, Barbara Hall. 1973. Opacity, coreference, and pronouns. In Semantics of natural language, pages 415-441. Springer.

Portner, Paul. 2009. Modality, vol. 1. Oxford University Press.

Roberts, Craige. 1987. Modal subordination, anaphora, and distributivity. Ph.D. thesis, University of Massachusetts Amherst.

Roberts, Craige. 1989. Modal subordination and pronominal anaphora in discourse. Linguistics and philosophy 12(6):683-721.

Schoubye, Anders J. 2011. Epistemic modality and natural language. University Lecture. Carnegie Mellon University.

Sells, Peter. 1985. Restrictive and non-restrictive modification, vol. 28. Center for the Study of Language and Information, Stanford University.

Simons, Mandy. 1996. Disjunction and anaphora. In Proceedings of SALT, vol. 6, pages $245-260$.

Starr, William B. 2012. Kratzer on modality in natural language. University Lecture. Cornell University.

Stone, Matthew. 1999. Reference to possible worlds. Tech. Rep. 49, Center for Cognitive Science, Rutgers University.

Strachey, Christopher and Christopher P. Wadsworth. 1974. Continuations: A mathematical semantics for handling full jumps. Tech. rep., Oxford University Computing Laboratory, Programming Research Group (Oxford).

Tarski, Alfred. 1944. The semantic conception of truth: and the foundations of semantics. Philosophy and phenomenological research 4(3):341-376.

Tarski, Alfred. 1956. The concept of truth in formalized languages. Logic, semantics, metamathematics 2:152-278.

von Fintel, Kai. 2006. Modality and language. In D. M. Borchert, ed., Encyclopedia of philosophy - second edition. Detroit : Macmillan Reference USA. 



\section{Appendix}

\section{Elaboration on Formal Details}

This appendix aims to unfold the formal details of definitions in section 4. We will start with the elementary functions in section 4.2, then we shall look into the lexical entries in section 4.4.

\section{Elementary Functions}

In the following context, we provide a description for each elementary function defined in section 4.2

\subsection{Modalized Logical Constants}

- Modal conjunction: $o_{i} \rightarrow o_{i} \rightarrow o_{i}$

$$
\wedge_{i} \triangleq \lambda A B i .(A i \wedge B i)
$$

The operator $\wedge_{i}$ is the modal counterpart of $\wedge$. It takes two modal propositions as input, and returns another modal proposition, which is the conjunction consisting of the logical contents in the input.

- Modal negation: $o_{i} \rightarrow o_{i}$

$$
\neg_{i} \triangleq \lambda A i \cdot \neg(A i)
$$

The operator $\neg_{i}$ is the modal counterpart of $\neg$. It takes a modal proposition as input, and returns its modal negation.

- Modal existential quantifier for individuals: $\left(\iota \rightarrow o_{i}\right) \rightarrow o_{i}$

$$
{ }^{\iota} \exists_{i} \triangleq \lambda P i .{ }^{\iota} \exists(\lambda x . P x i)
$$

The operator ${ }^{\iota} \exists_{i}$ is the modal counterpart of ${ }^{\iota} \exists$. It takes a modal individual property (of type $\iota \rightarrow o_{i}$ ) as input, and returns an existentially quantified modal proposition.

- Modal tautology: $o_{i}$

$$
\top_{i} \triangleq \lambda i . \top
$$

The tautology $T$ is of type $o$, it always denotes the truth value 1. Its counterpart in modal systems: $\top_{i}$, which returns 1 at each 
possible world, is of type $o_{i}$.

\subsection{Environment and Salient World Manipulation}

- Retrieve the salient world: $\gamma_{i} \rightarrow s$

$$
\text { woi } \triangleq \lambda e . \pi_{1} e
$$

The function woi is relatively straightforward. It takes a left context $e$ as input and returns its salient world, which is simply the first projection of $e$.

- Retrieve the generalized environment: $\gamma_{i} \rightarrow T_{g e n v}$

$$
\text { genv } \triangleq \lambda e \cdot \pi_{2} e
$$

In contrast to the previous function woi, the function genv takes a left context $e$ and returns its generalized environment, which corresponds to the second projection of the input $e$.

- Retrieve the environment: $\gamma_{i} \rightarrow s \rightarrow T_{\text {env }}$

$$
\text { env } \triangleq \lambda e i .(\text { genv } e i)
$$

The function env is established upon genv (formula 4.13). It takes a left context and a possible world, and returns a specific environment at the input world.

- Modify the salient world: $\gamma_{i} \rightarrow s \rightarrow \gamma_{i}$

$$
\text { change_woi } \triangleq \lambda e i .\langle i,(\text { genv } e)\rangle
$$

The function change_woi takes a left context $e$ and a possible world $i$ as input. It yields a new left context, where the salient world is modified to the input world $i$, the generalized environment is the one of the input left context.

- Retrieve the background: $\gamma_{i} \rightarrow s \rightarrow o_{i}$

$$
\mathbf{b k g d} \triangleq \lambda e i . \pi_{1}(\mathbf{e n v} e i)
$$

The function bkgd takes a left context $e$ (a Cartesian product consisting of a salient world and a generalized environment) and a possible world $i$ as input. It yields a modal proposition, which is the background (the first element of the environment) of the left context $e$ at the given world $i$.

- Retrieve the base: $\gamma_{i} \rightarrow s \rightarrow o_{i}$

$$
\text { base } \triangleq \lambda e i . \pi_{2}(\mathbf{e n v} \text { e } i)
$$

The function base takes a left context $e$ (a Cartesian product consisting of a salient world and a generalized environment) and a possible world $i$ as input. It yields a modal proposition, which is the base (the second element of the environment) of the left context $e$ at the given world $i$. 


\subsection{Context Manipulation}

- Update the generalized environment: $T_{\text {genv }} \rightarrow s \rightarrow T_{\text {env }} \rightarrow T_{\text {genv }}$

$$
\text { up_genv } \triangleq \lambda G i E . G[i:=E]
$$

The function up_genv takes three arguments as input:

1.A generalized environment $G$, which is of type $T_{g e n v}$;

2.A possible world $i$, which is of type $s$, it denotes the target world at which the generalized environment is to be updated;

3.A to-be-updated environment $E$, which of type $T_{e n v}$.

It thus yields another generalized environment, namely $G[i:=E]$.

- Update the left context: $\gamma_{i} \rightarrow s \rightarrow o_{i} \rightarrow \gamma_{i}$

$$
\begin{aligned}
\text { up_context } \triangleq \lambda e i A . & \langle(\text { woi } e), \\
& \text { up_genv } \\
& (\text { genv } e) \\
& i \\
& \left\langle A \wedge_{i}(\text { bkgd } e i), A \wedge_{i}(\text { base } e i)\right\rangle \\
\rangle &
\end{aligned}
$$

The function up_context takes three arguments as input:

1.A left context $e$, which is of type $\gamma_{i}$;

2.A possible world $i$ at which the update process takes place, it is of type $s$;

3.A modal proposition $A$, which is the to-be-updated logical content, it is of type $o_{i}$.

It yields an updated left context, with the logical content of the modal proposition $A$ added in both the background and the base of $e$ at world $i$.

- Copy the left context: $\gamma_{i} \rightarrow s \rightarrow s \rightarrow \gamma_{i}$

$$
\begin{array}{r}
\text { copy_context } \triangleq \lambda e i j .\langle(\text { woi } e), \\
\text { up_genv }
\end{array}
$$

$$
\begin{aligned}
& (\operatorname{genv} e) \\
& j \\
& (\operatorname{env} e i)
\end{aligned}
$$

The function copy_context takes a left context $e$ and two possible worlds $i$ and $j$ as input. It yields a left context, which has the same salient world as the input left context, but the original environment at world $i$ will be copied to all worlds that are accessible from $j$. 
- Reset the base in a left context: $\gamma_{i} \rightarrow s \rightarrow \gamma_{i}$

$$
\text { reset_base } \triangleq \lambda e i .\langle(\text { woi } e),
$$

$(\operatorname{genv} e)$
$i$

$\left\langle(\right.$ bkgd $\left.e i), \top_{i}\right\rangle$

During the discourse processing, we will have to reset the base at various steps (particularly, when the proposition in the base has already been used) in order to avoid information duplication. The above function reset_base helps to achieve this goal. Basically, reset_base takes a left context $e$ and a possible world $i$ as input. It yields another left context, which contains the same salient world, and a modified generalized environment, where the base information is reset.

- The empty left context: $\gamma_{i}$

$$
\operatorname{nil}_{i} \triangleq\left\langle\mathbf{H}, \lambda i \cdot\left\langle\top_{i}, \top_{i}\right\rangle\right\rangle
$$

The term nil ${ }_{i}$ represents the void left context in M-TTDL. It is a context at the current world $\mathbf{H}$, and both background and base propositions in the environment are the modal tautology $T_{i}$. It is similar to the nil in TTDL.

- The empty right context: $\gamma_{i} \rightarrow o_{i}$

$$
\operatorname{stop}_{i} \triangleq \lambda e \cdot \top_{i}
$$

Analogous to the term stop (formula 2.1) in previous frameworks, the above term stop sis $_{i}$ an empty right context in M-TTDL. It takes a left context as input, no matter what its value is, it always returns the modal tautology $\top_{i}$. As discussed in section 4.1, the way that the context is unfolded is rather complex in M-TTDL. Thus at the end of the discourse processing, stop $_{i}$ may be employed together with nil ${ }_{i}$ in order to obtain a more concise and compact logical representation. We see its application in section 4.6

\section{Lexical Entries for Modals}

This part of the appendix aims to give a detailed explanation for the lexical entries defined in section 4.4. 


\subsection{Possibility Modal Operator}

The entry of the modal operator $\diamond$ is defined as follows:

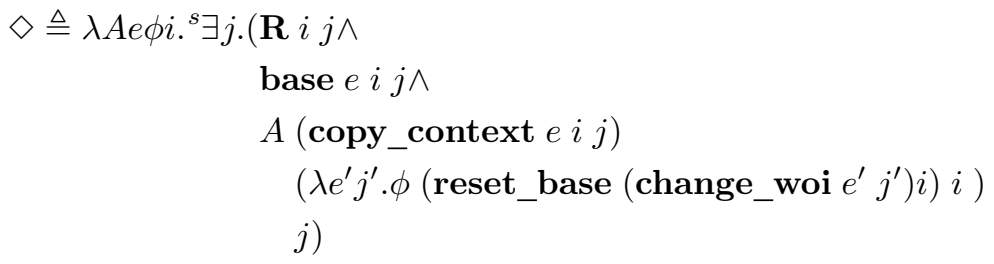

The above entry can be understood as follows. The quantifier ${ }^{s} \exists$ ranges over possible world variables, $\mathbf{R}$ denotes the accessibility relation, so ${ }^{s} \exists j . \mathbf{R} i j$ means there exists a possible world $j$ which is accessible from world $i$. The modal base at world $i$, namely, the proposition that is necessarily true at the utterance world, should be satisfied in all of its accessible worlds, including $j$. This explains the sub-part base $e i j$, which serves as the common background in world $j$. As to the input proposition $A$, it is first applied to a left context, where the environment at the utterance world $i$ is copied to the newly established possible world $j$, this corresponds to copy_context $e i j$. Then, a right context, where the base at world $i$ is reset to the modal tautology $\top_{i}$, and the world of interest is switched to world $j$, is passed to $A$. Finally, the input proposition is evaluated at world $j$.

In conclusion, a dynamic proposition $A$ is possibly true, namely $\diamond A$ is satisfied, iff there is a possible world $j$, which is accessible from the utterance world $i$, such that:

- The propositions which are necessarily true at world $i$, namely the modal base at $i$, should be satisfied at world $j$;

- The possible world $j$ inherits the generalized environment from the utterance world $i$, and the base information at the utterance world $i$ is reset to $\top_{i}$;

- The salient world is updated to world $j$;

- The modalized proposition is evaluated at world $j$.

Now let's have a look at an example. Assume $A$ is a dynamic proposition (of type $\Omega_{i}$ ), where no modality is involved, $a$ is the logical content of $A$ (of type $o_{i}$ ). That is to say, $A$ is constructed by translating $a$ with respect to the dynamization rules, see definition 4.5. In order to illustrate how the above entry of $\diamond$ works, we shall contrast the environment of proposition $A$ and $\diamond A$.

Assume $\mathbf{m b}$ is the modal base function, which returns the background information at a given world. For more detail, please refer back 
to Kratzer's theory in section 3.2. The possible worlds hierarchy for interpreting the dynamic modal proposition $A$ is presented in figure 1 , where circles are used to denote possible worlds, a solid line with an arrow indicates the accessibility relation, a dotted line means the accessibility relation is not specified. Besides, we use the grey color to signify the salient world, assume the current left context is $e$, we will term the salient world $w_{s}$, namely $w_{s}=$ woi $e$. For the world of utterance, we uniquely term it $i$. Finally, we place the propositions that are true at each world besides it, e.g., $A$ is besides world $i$ in figure 1 . In subsequent diagrams, we will use the same notation style.

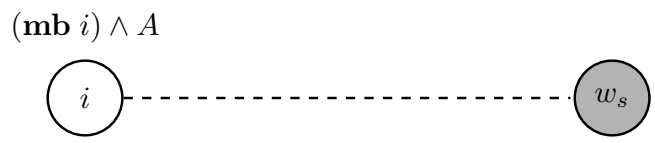

FIGURE 1 Possible Worlds Hierarchy of $A$

By default, proposition $A$ is uttered at world $i$. Because $A$ is not concerned with any modality, it is true iff $A$ is satisfied at $i$. Remark that $(\mathbf{m b} i) \wedge A$ and $A$ have the same truth conditions, because $(\mathbf{m b} i)$ is already satisfied at $i$. As shown in figure 1 the interpretation of $A$ will not change the salient world. In addition, since we do not have further information on the relation between the utterance world $i$ and the salient world $w_{s}$, their accessibility is unspecified. Table 1 lists the detailed content of the environment at each possible world in question:

\begin{tabular}{|c|c|}
\hline Existing World & Environment \\
\hline \hline$i$ & $\left\langle a \wedge_{i}(\right.$ bkgd $e i), a \wedge_{i}($ base $\left.e i)\right\rangle$ \\
\hline$w_{s}$ & $\langle($ bkgd $e($ woi $e)),($ base $e($ woi $e))\rangle$ \\
\hline
\end{tabular}

TABLE 1 Environment at Each World of $A$

To sum up, after the interpretation of $A$, both elements in the environment at world $i$, namely the background and base are updated with $a$; while the environment at the salient world $w_{s}$ is not modified.

Let's turn to $\diamond A$, its possible worlds hierarchy is depicted in figure 2 .

Again, the proposition $\diamond A$ is uttered at world $i$. $A$ is possibly true at the utterance world $i$, or equivalently, $\diamond A$ is true at $i$, iff $(\mathbf{m b} i) \wedge$ $A$ is true at an accessible world from $i$, e.g., $j$, where $(\mathbf{m b} i)$ is the background information at $i$. As presented above, the lexical entry of 


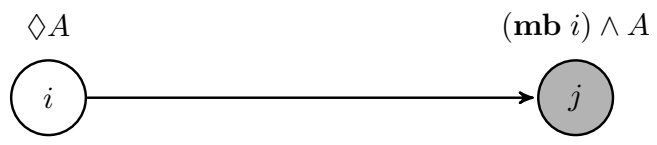

FIGURE 2 Possible Worlds Hierarchy of $\diamond A$

$\diamond$ modifies the salient world to the newly established world $j$. The detailed content of environment at each world is listed in table 2 .

\begin{tabular}{|c|c|}
\hline Existing World & Environment \\
\hline \hline$i$ & $\left\langle(\right.$ bkgd $\left.e i), \top_{i}\right\rangle$ \\
\hline$j$ & $\left\langle a \wedge_{i}(\right.$ bkgd $e i), a \wedge_{i}($ base $\left.e i)\right\rangle$ \\
\hline
\end{tabular}

TABLE 2 Environment at Each World of $\diamond A$

The interpretation of $\diamond A$ requires an accessible possible world from the utterance world, in which $A$ is satisfied. Furthermore, its logical content $a$ is updated to the environment of the salient world. At the same time, the base information at the evaluation world is reset to a modal tautology to avoid information duplication.

\subsection{Necessity Modal Operator}

The entry of the modal operator $\square$ is defined as follows:

$\square \triangleq \lambda \operatorname{Ae\phi i.}\left({ }^{s} \forall j .(\mathbf{R} i j \rightarrow\right.$

$$
\begin{aligned}
& (\text { base } e i j \rightarrow \\
& \left.\left.\left.\qquad\left(A(\text { copy_context } e i j) \text { stop }_{i} j\right)\right)\right)\right)
\end{aligned}
$$

$\wedge \phi e i$

The above entry can be understood as follows. The quantifier ${ }^{s} \forall$ ranges over possible world variables, so ${ }^{s} \forall j$.R $i j$ means for every possible world $j$ that is accessible from the utterance world $i$. The modal base at world $i$, namely, the proposition that is necessarily true at the utterance world, should be satisfied in all of its accessible worlds. This explains the sub-part base $e i j$. Different from in $\diamond$, where the modal base is conjuncted, it plays the role of antecedent of an implication in the necessity modality. As to the input proposition $A$, similar to $\diamond$, it is first applied to a left context, where the environment at the utterance world $i$ is copied to the newly established possible world $j$, this corresponds to copy_context $e i j$. However, due to the semantics of the necessity modality, referents introduced in the scope of $\square$ shall not be 
accessed from subsequent context. As a result, the empty continuation stop $_{i}$ is passed to $A$, and the current left context will not be modified after processing the modalized proposition.

In conclusion, a dynamic proposition $A$ is necessarily true, namely $\square A$ is satisfied, iff for every possible world $j$, if $j$ is accessible from the utterance world $i$, then:

- The propositions which are necessarily true at world $i$, namely the modal base at $i$, should be satisfied and serve as premise assumption at world $j$;

- The possible world $j$ inherits the generalized environment from the utterance world $i$;

- Information at every possible world $j$ cannot to be passed to subsequent sentences;

- The left context is not changed, the utterance world of the modalized proposition is still $i$, and proposition $A$ is evaluated at world $j$.

Now we turn to an example. Like $\diamond A, \square A$ is also established upon $A$. We may compare the following information with the one presented in figure 1 and table 1 . The possible worlds hierarchy of interpreting $\square A$ is illustrated in figure 3

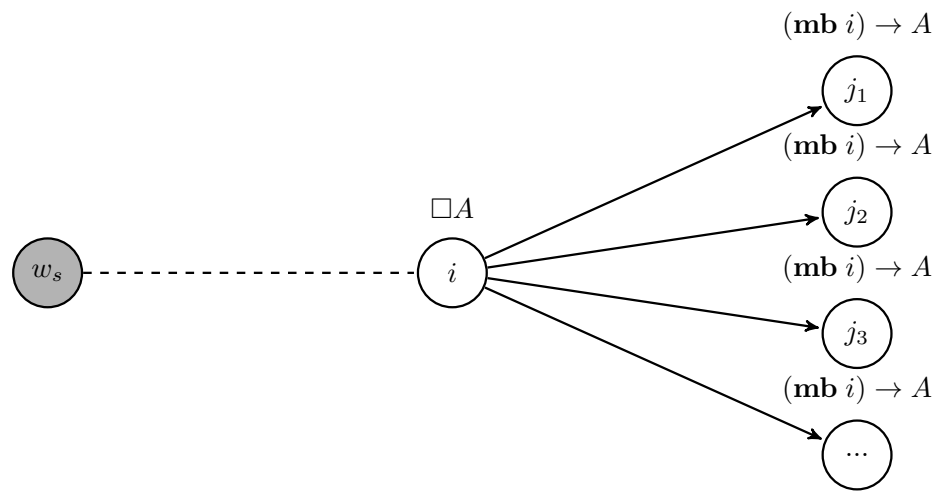

FIGURE 3 Possible Worlds Hierarchy of $\square A$

Same as above, the utterance world is $i$. $A$ is necessarily true at the utterance world $i$, or equivalently, $\square A$ is true at $i$, iff $(\mathbf{m b} i) \rightarrow A$ is true at every accessible world from $i$, where $(\mathbf{m b} i)$ is the background information at $i$. As discussed above, the lexical entry of $\square$ does not modify the salient world, which is still the original $w_{s}$. Also, the relation 
between the salient world and the utterance world is not specified. For the detailed content of environment at each possible world, we can refer to table 3 .

\begin{tabular}{|c|c|}
\hline Existing World & Environment \\
\hline \hline$i$ & $\langle($ bkgd $e i),($ base $e i)\rangle$ \\
\hline$j$ & $\langle($ bkgd $e j),($ base $e j)\rangle$ \\
\hline$w_{s}$ & $\langle($ bkgd $e($ woi $e)),($ base $e($ woi $e))\rangle$ \\
\hline
\end{tabular}

TABLE 3 Environment at Each World of $\square A$

After interpreting a necessity modality, the environment at every existing world, such as the salient world $w_{s}$, the utterance world $i$, and all its accessible worlds $j$, remain unchanged. Hence the operator $\square$ does not modify the context change potential of the preceding discourse. On the one hand, it does not change the salient world; on the other hand, it does not modify the context at any possible world.

\subsection{Evaluation "at" Some Possible World}

The entry of the at function is defined as follows:

$$
\begin{aligned}
& \text { at } \triangleq \lambda j A e \phi i \\
& \text { if }(j=i) \\
& (A e \phi i) \\
& (\text { base } e i j \wedge \\
& A(\text { up_context } e j(\text { base } e i)) \\
& \left(\lambda e^{\prime} j^{\prime} . \phi\left(\text { reset_base } e^{\prime} i\right) i\right) \\
& j)
\end{aligned}
$$

In the above formula, the two input arguments, $j$ and $A$, stand for the target world of evaluation and the dynamic proposition to be evaluated, respectively. The rest of the entry can be understood as follows. Firstly, the operator if is a logical constant, it is used to determine whether the target world $j$ is identical to the current utterance world $i$ or not. If the two worlds happen to coincide, the second argument will be returned. No modification is needed in this case: the proposition is by default evaluated in the utterance world. Otherwise, if the proposition is to be evaluated in another world than the utterance world $i$, the third argument will be returned. In this case, the base at $i$ is updated to the context of the target world $j$ by the context update function (we do not use the function copy_context because it will overwrite the environment at world $j$ ). Furthermore, as for $\diamond$, after employing the 
modal base at world $i$, we reset it as the modal tautology, this explains the sub-part reset_base $e^{\prime} i$.

In conclusion, a dynamic proposition $A$, which is uttered at world $i$, is interpreted true at another possible world $j$, iff

- The base proposition at the utterance world $i$ is passed to the target world $j$;

- The context at world $j$ is updated with the base proposition from world $i$;

- The base of the utterance world $i$ is reset after being employed;

- The logical content of proposition $A$ is evaluated at the target world $j$.

Finally, as above, we provide an illustration, which elucidates the environment of at $\mathbf{H} A$, where $\mathbf{H}$ is a possible world constant, $A$ is the dynamic proposition to be evaluated. Again, we assume $a$ is the logical content of $A$. Since at $\mathbf{H} A$ is built upon $A$, we may contrast the following analysis with the information in figure 1 and table 1, which will not be repeated here any more.

First of all, the possible worlds hierarchy of at $\mathbf{H} A$ is illustrated in figure 4 .

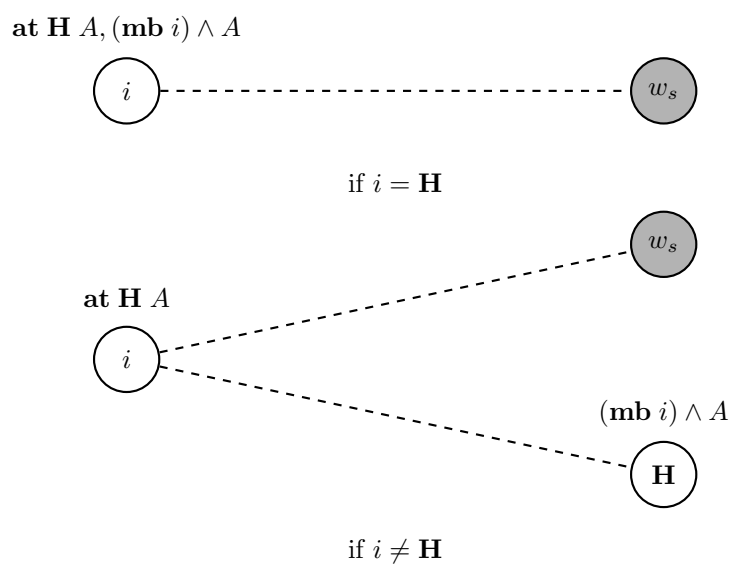

FIGURE 4 Possible Worlds Hierarchy of at $\mathbf{H} A$

We have to distinguish two cases: if the utterance world $i$ is equal to the target world $\mathbf{H}$, at $\mathbf{H} A$ and $A$ are identical formulas, that is to say, at $\mathbf{H} A$ being true at $\mathbf{H}$ is equivalent to $A$ being true at $i$, as shown in 
the upper part of figure 4 , otherwise, if $i$ and $\mathbf{H}$ are different worlds, at $\mathbf{H} A$ is true at $i$ means $(\mathbf{m b} i) \wedge A$ is true at $\mathbf{H}$, where $(\mathbf{m b} i)$ is the background information at the utterance world $i$, as shown in the lower portion of figure 4. The at function merely evaluates a dynamic proposition at another world, it does not change the default salient world $w_{s}$. Also no explicit accessibility relation among possible worlds, such as between $i$ and $w_{s}$, between $i$ and $\mathbf{H}$, can be induced from at.

As to the detailed content of the environment at each world, we will also have to distinguish the above mentioned two cases. These two situations are listed separately in table 4

\begin{tabular}{|c|c|c|}
\hline \multicolumn{2}{|c|}{ Existing World } & Environment \\
\hline \hline \multirow{2}{*}{$i=\mathbf{H}$} & $\mathbf{H}$ & $\left\langle a \wedge_{i}(\right.$ bkgd $e i), a \wedge_{i}($ base $\left.e i)\right\rangle$ \\
\cline { 2 - 3 } & $w_{s}$ & $\langle($ bkgd $e($ woi $e)),($ base $e($ woi $e))\rangle$ \\
\hline \hline \multirow{3}{*}{$i \neq \mathbf{H}$} & $i$ & $\left\langle(\right.$ bkgd $\left.e i), \top_{i}\right\rangle$ \\
\cline { 2 - 3 } & $w_{s}$ & $\langle($ bkgd $e($ woi $e)),($ base $e($ woi $e))$ \\
\cline { 2 - 3 } & $\mathbf{H}$ & $\begin{array}{c}\left\langle a \wedge_{i}(\text { base } e i) \wedge_{i} \operatorname{bkgd}(e, \mathbf{H}),\right. \\
\left.a \wedge_{i}(\text { base } e i) \wedge_{i} \text { base }(e, \mathbf{H})\right\rangle\end{array}$ \\
\hline
\end{tabular}

TABLE 4 Environment at Each World of at $\mathbf{H} A$

On the one hand, when the target world $\mathbf{H}$ is identical to the utterance world $i$, the result of at $\mathbf{H} A$ is the same as the one for dynamic proposition $A$, see table 1 . This is exactly what we expect. On the other hand, when $\mathbf{H}$ is different from $i$, the base proposition of $i$, together with the logical content of $A$, will be updated to the environment of $\mathbf{H}$.

\subsection{Modal Expressions}

The lexical entry of the modal verb might is defined as follows:

$$
\llbracket m i g h t \rrbracket_{M-T T D L}=\lambda A e \phi i .(\text { at }(\text { woi } e)(\diamond A)) e \phi i
$$

The unfolding of 4.31 is rather tedious, we will not do it here. Instead, as previously, we will provide the possible worlds hierarchy and the environment status of $\llbracket m i g h t \rrbracket_{M-T T D L} A$ as an illustration. Since might involves function at, we will have to determine the identity between the salient world $w_{s}$ and the utterance world $i$. The following analysis, which will be divided into two separate cases, is analogous to the one for at. In addition, since $\llbracket m i g h t \rrbracket_{M-T T D L} A$ is built upon $A$, we can compare the following analysis with the information in figure 1 and table 1. which will not be repeated here any more.

The possible worlds hierarchy of interpreting $\llbracket m i g h t \rrbracket_{M-T T D L} A$ is illustrated in figure 5 in which we abbreviate $\llbracket m i g h t \rrbracket_{M-T T D L} A$ as $\mathbf{M} A$. 
Its environment status is listed in table 5
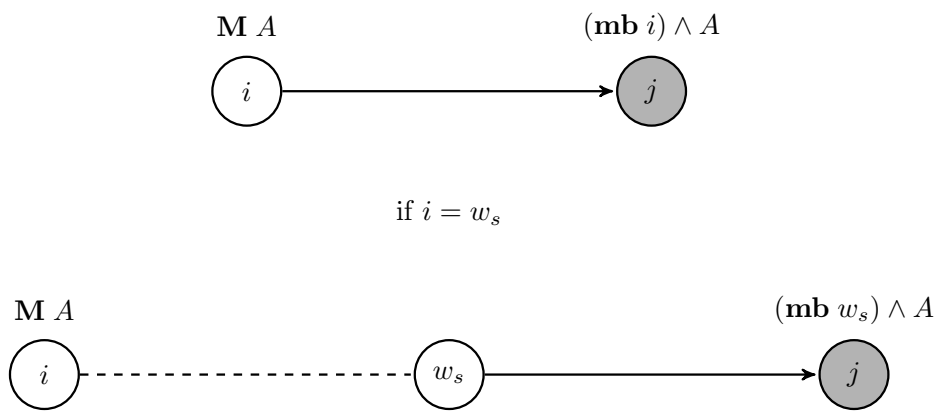

if $i \neq w_{s}$

FIGURE 5 Possible Worlds Hierarchy of $\llbracket m i g h t \rrbracket_{M-T T D L} A$

\begin{tabular}{|c|c|c|}
\hline \multicolumn{2}{|c|}{ Existing World } & Environment \\
\hline \hline \multirow{2}{*}{$i=w_{s}$} & $i$ & $\left\langle(\right.$ bkgd $\left.e i), \top_{i}\right\rangle$ \\
\cline { 2 - 3 } & $j$ & $\left\langle a \wedge_{i}(\right.$ bkgd $e i), a \wedge_{i}($ base $\left.e i)\right\rangle$ \\
\hline \hline \multirow{3}{*}{$i \neq w_{s}$} & $i$ & $\left\langle(\right.$ bkgd $\left.e i), \top_{i}\right\rangle$ \\
\cline { 2 - 3 } & $w_{s}$ & $\left\langle(\right.$ base $e i) \wedge_{i}($ bkgd $e($ woi $\left.e)), \top_{i}\right\rangle$ \\
\cline { 2 - 3 } & $j$ & $\begin{array}{c}\left\langle a \wedge_{i}(\text { base } e i) \wedge_{i}(\text { bkgd } e(\text { woi } e)),\right. \\
\left.a \wedge_{i}(\text { base } e i) \wedge_{i}(\text { base } e(\text { woi } e))\right\rangle\end{array}$ \\
\hline
\end{tabular}

TABLE 5 Environment at Each World of $\llbracket m i g h t \rrbracket_{M-T T D L} A$

If we compare the effect of $\llbracket m i g h t \rrbracket_{M-T T D L}$ with $\diamond$, namely figure 5 with figure 2 , table 5 with table 2 , we can find out that $\llbracket m i g h t \rrbracket_{M-T T D L} A$ and $\diamond A$ generate the same result when the default salient world $w_{s}$ is identical to the utterance world $i$; however, if the two worlds are different, $\llbracket m i g h t \rrbracket_{M-T T D L} A$ and $\diamond A$ will generate different results. This is because when interpreting $\llbracket m i g h t \rrbracket_{M-T T D L} A$, a new possible world $j$ will be established over the salient world $w_{s}$, rather than over the utterance world $i$, as shown in the lower part of figure 5

The semantic entry for the modal verb would is defined as follows:

$$
\llbracket \text { would } \rrbracket_{M-T T D L}=\lambda A e \phi i .(\text { at }(\text { woi } e)(\square A)) e \phi i
$$

Likewise, we will not unfold 4.32 but an analogous illustration con- 
taining the possible worlds structure and the environment status will be provided. As $\llbracket m i g h t \rrbracket_{M-T T D L} A, \llbracket w o u l d \rrbracket_{M-T T D L} A$ is also built upon $A$, we can compare the following analysis with the information in figure 1 and table 1 .

The possible worlds hierarchy of interpreting $\llbracket w o u l d \rrbracket_{M-T T D L} A$ is illustrated in figure 6 in which we abbreviate $\llbracket w o u l d \rrbracket_{M-T T D L} A$ as $\mathbf{W} A$ :

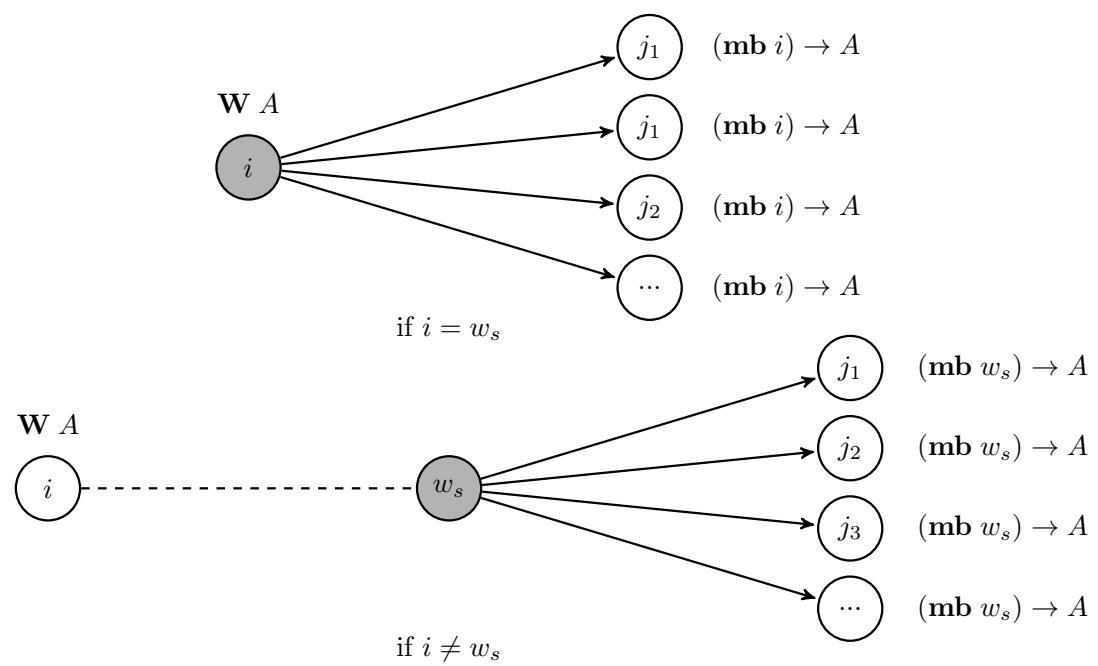

FIGURE 6 Possible Worlds Hierarchy of $\llbracket w o u l d \rrbracket_{M-T T D L} A$

The environment status for $\llbracket$ would $\rrbracket_{M-T T D L} A$ is listed in table 6 . The two situations, one where $i$ equals $w_{s}$, and the other where they are different, are separately presented.

\begin{tabular}{|c|c|c|}
\hline \multicolumn{2}{|c|}{ Existing World } & Environment \\
\hline \hline \multirow{2}{*}{$i=w_{s}$} & $i$ & $\langle($ bkgd $e i),($ base $e i)\rangle$ \\
\cline { 2 - 3 } & $j$ & $\langle($ bkgd $e j),($ base $e j)\rangle$ \\
\hline \hline \multirow{3}{*}{$i \neq w_{s}$} & $i$ & $\left\langle(\right.$ bkgd $\left.e i), \top_{i}\right\rangle$ \\
\cline { 2 - 3 } & $w_{s}$ & $\begin{array}{c}\left\langle(\text { base } e i) \wedge_{i}(\text { bkgd } e(\text { woi } e)),\right. \\
\left.(\text { base } e i) \wedge_{i}(\text { base } e(\text { woi } e))\right\rangle\end{array}$ \\
\cline { 2 - 3 } & $j$ & $\langle($ bkgd $e j),($ base $e j)\rangle$ \\
\hline
\end{tabular}

TABLE 6 Environment at Each World of $\llbracket$ would $\rrbracket_{M-T T D L} A$

As motivated by the comparison between might and $\diamond$, a cor- 
responding contrast can be drawn between $\llbracket w o u l d \rrbracket_{M-T T D L}$ and $\square$ : $\llbracket$ would $\rrbracket_{M-T T D L} A$ and $\square A$ yield the same result when the default salient world $w_{s}$ is identical to the utterance world $i$, see figure 6 and figure 3 . table 6 and table 3 , while when the two worlds are different, the dynamic proposition $\square A$ is still uttered at world $i$, but since the salient world $w_{s}$ is not $i$, possibly due to some modality in the preceding discourse, the necessity from $\square A$ will be established over $w_{s}$, rather than over $i$.

The entries for might and would share many properties, especially the way they affect the possible worlds hierarchy in different situations. However, as we may notice at the same time, $\llbracket m i g h t \rrbracket_{M-T T D L}$ has the potential to update the salient world, while $\llbracket$ would $\rrbracket_{M-T T D L}$ does not. In addition, $\llbracket m i g h t \rrbracket_{M-T T D L}$ always resets the base of the salient world, the one based on which new worlds are built upon, while $\llbracket$ would $\rrbracket_{M-T T D L}$ does not. 